\title{
19. THE MEASUREMENT OF INCOME MOBILITY: AN INTRODUCTION TO THE LITERATURE
}

\author{
Gary S. Fields and Efe A. Ok ${ }^{1}$
}

\section{Introduction}

Evaluation of distributions of income on the basis of equality is one of the most extensively studied topics in the general field of welfare economics. There is thus an enormous body of work devoted exclusively to the problem of the measurement of welfare and income inequality experienced by a given society. ${ }^{2}$ However, the majority of these studies are conducted in static terms, where the so-called "snapshots" of the income distributions are taken as the primitives of the analysis. Nevertheless, static evaluations of income distributions can provide only an incomplete picture, for, in most instances, the social welfare would certainly depend on the dynamics of income distribution as well. ${ }^{3}$ This basic insight, along with the increased availability of longitudinal data sets, has led to a massive and rapidly expanding literature on the measurement of income mobility. This chapter surveys the highlights of this literature.

In contrast to income inequality, "income mobility" concerns the changes in economic status from one time period or generation to another. ${ }^{4}$ Consequently, mobility studies take time paths of income distributions as the primitives of the analysis, and in this sense, are of multivariate nature. These studies usually take place either in intragenerational contexts by means of longitudinal or retrospective data for the same individual, or in intergenerational contexts where the focus is rather on the relationship between the incomes of the parents and of their offspring.

There is, indeed, a substantial literature on how to measure income mobility. But unlike the theories of measurement of income inequality and economic poverty, the mobility literature does not provide a unified discourse of analysis. This might be because the very notion of income mobility is not well-defined; different studies concentrate on different aspects of this multi-faceted concept. At any rate, it seems safe to say that a considerable degree of confusion confronts a newcomer to the field. 
Our main purpose in this survey is, therefore, to provide a somewhat unified setting within which the basic features of the theory of income mobility measurement can be outlined. Our aim is to provide a (subjectively) selective introduction to the literature on income mobility, and thereby shed some light on particular aspects of mobility measurement. Consequently, the present study should not be viewed as an exhaustive survey of the related literature. It is rather a very concise account of some key elements of the theory of income mobility measurement. ${ }^{5}$

The organization of this chapter is as follows. In section 2 , we introduce a simple framework for analysis which allows us to examine a number of fundamental issues concerning our perceptions of "income mobility". In this introductory section, we try to illustrate the distinctions between some key aspects of the mobility concept including the notions of temporal independence and aggregate income movements. Particular attention is given to the examination of some elementary concepts like transition matrices, relative vs. absolute mobility, and exchange vs. structural mobility.

There are only few axiomatic studies on the measurement of income mobility; a situation which stands in stark contrast with the sister measurement theory of income inequality. Section 3 shows that axiomatics may, in fact, turn out to be very fruitful in mobility measurement. In this section, we characterize a number of measures of income movement which may prove useful in future empirical work. One of these indices is even additively decomposable into two parts, and this yields a practical disaggregation of total mobility into growth and transfer components. Finally, a descriptive income mobility ordering, which is reminiscent of the celebrated Lorenz ordering in some respects, is also introduced in section 3.

Section 4 outlines a number of welfarist approaches developed in the context of income mobility measurement in recent years, and studies the mobility indices of King and Chakravarty, Dutta and Weymark. These measures not only exhibit the general features of a welfarist mobility index, but also broaden one's ethical perspective by addressing two distinct normative facets of income mobility. Finally, in section 4, we present a brief discussion of Atkinson's well-known normative mobility ordering, and a new index of relative income mobility. 
In section 5, we turn to a different setting which is suitable for introducing perhaps the most popular approach in income mobility measurement, namely, the Markovian approach. This framework proves particularly useful in clarifying the difference between the fundamental mobility notions of temporal independence and aggregate income movements. Next, we illustrate how the performance of the Markovian approach is improved by the recent introduction of monotone mobility matrices. In particular, we present a simple example which demonstrates explicitly that monotone Markov chains have a lot to say on the issue of intertemporal inequality dominance. Finally, we introduce Dardanoni's (social) mobility ordering, briefly discuss some of its properties, and close section 5 with a caveat outlining some conventional criticisms of the basic assumptions of the Markovian mobility model. Our survey concludes with a few final remarks.

\section{Basic Concepts of Mobility Measurement}

\subsection{A framework for analysis}

The basic primitive of any study on income mobility is the time path of the income distribution among the same individuals or among the dynasties in a given society. In its simplest form, therefore, such a study requires at least two observations on income. To make things precise, let us adopt the convention of taking $R_{+}^{n}$ as the space of all income distributions with population $n \geq 1$. Consequently, $x=\left(x_{1}, \ldots, x_{n}\right) \in R_{+}^{n}$ represents the income distribution of an n-person society where agent $i$ 's income level is $x_{i}$. Now assume that the ith agent's income has changed to $y_{i}$ in a given time interval. We say that $x$ has been transformed to $y=\left(y_{1}, \ldots, y_{n}\right) \in R_{+}^{n}$, and denote this so-called distributional transformation by $x \rightarrow y$. Distilled to its simplest form, the theory of income mobility measurement can be defined as the study of these transformations.

While it admittedly confines the analysis to only 2-period paths of income distributions, this framework allows one to study both intra and intergenerational mobility measurement, depending on the length of the time period between the observation periods. In view of the fact that some studies on mobility measurement take the number of observation periods into consideration in a crucial manner, the present formulation is thus admittedly not without loss of generality. ${ }^{6}$ As we shall see, however, it allows one to introduce most of the key concepts of mobility measurement theory, and is thus useful with respect to our expositional purposes. 
In passing, we note that a large number of studies on income mobility are, in fact, conducted directly in terms of the stochastic processes assumed to generate the observed path of income distributions. Our simple framework is, of course, not suitable to analyze what can and cannot be achieved in such probabilistic settings. We shall, therefore, consider a conventional stochastic framework as well later in the survey.

\subsection{Income mobility measures}

Given the framework described above, we define a mobility measure (index) as any continuous function $f: R_{*}^{2 n} \rightarrow R$ with the interpretation that " $x \rightarrow y$ exhibits more mobility (as measured by $f$ ) than $z \rightarrow w$ whenever $f(x, y) \geq f(z, w)$ ". (For simplicity, we do not adopt a notation in this section which would make the dependence of $f$ on $n$ explicit.) It is not readily clear how to improve upon this disturbingly general definition before clarifying what one really means by "income mobility". But clarifying the meaning of mobility turns out to be quite non-trivial. Indeed, we shall observe throughout this survey that the very notion of income mobility is rather a multi-faceted one, and any attempt to devise a measure that aims to incorporate all aspects of income mobility is therefore destined for failure.

To present a brief illustration of the intrinsic multi-dimensionality of the notion of income mobility, let us consider the following transformations:

$$
\begin{aligned}
& \text { I: } \quad x \equiv(1,3)-(1,3) \\
& \text { II: } x \equiv(1,3)-(3,1) \equiv y \\
& \text { III: } x \equiv(1,3) \rightarrow(2,2) \equiv z
\end{aligned}
$$

The question is: which of these distributional transformations depicts more mobility than the others?

In answering this question, many mobility analysts might choose to base their decisions on the correlation between the initial income distribution and the final one. Some might even use a simple mobility index like $f_{r}(x, y) \equiv 1-r(x, y)$ where $r(x, y)$ stands for the correlation coefficient between $x$ and $y$ (cf. Department of Employment, 1973). ${ }^{7}$ The measure $f_{r}$, and in fact all correlation-based indices (footnote 7) would say that process $I$ is certainly the least mobile of all: $f_{r}(x, x) \equiv 0$. We may easily think of at least two reasons for why this conclusion might be regarded as "obvious". First, no one's income has changed in process I, so nobody has moved away from the status quo. Second, the transformation seems very rigid in the sense that the final period income of a person is completely determined by his 
or her initial income level (or that of the parent(s)). In other words, there appears to be a perfect (positive) dependence of one's final income state on her initial state.

Unfortunately, these intuitions do not always agree. For instance, in comparing the processes II and III, one may argue that II is more mobile than III because in the former transformation we observe larger personal income changes than the latter; people simply move less in process III than in II. Indeed, $f_{r}$ confirms this intuition: $f_{r}(x, y) \equiv 2>1=f_{r}(x, z)$. There is a sense however in which III is more mobile than II since there appears to be no dependence of one's final income state on the initial state in process III, while we observe a perfect (negative) dependence in the case of process II. Therefore, $f_{r}$ fails to capture this origin independence aspect of the notion of income mobility, while it fares quite well with the movement aspect of mobility (see subsection 5.1 for more on this). For the purposes of measuring origin independence, one might prefer rather a mobility measure like $f_{|r|}(x, y) \equiv 1-|r(x, y)| .^{8}$

There are several reasons why a welfare economist would be interested in these facets of income mobility. In an intergenerational context, origin independence seems to capture our intuitions about "equality of opportunity" which can be roughly defined as the extent to which personal characteristics (like talent) rather than parental background determine monetary rewards (cf. Loury, 1981, Benabou, 1996 and Benabou and Ok, 1999). Viewed this way, income mobility is a desirable notion that helps attenuate the unequal distribution of initial endowments. Yet, in an intragenerational context, the movement aspect of mobility appears to be related to the notion of "lifetime income inequality". Indeed, process II is intertemporally more equal than process III in the sense of lifetime equality. ${ }^{9}$ What is more, the movement aspect of mobility relates directly to the income flux that takes place in the society. Thus, by using measures of income movement, one can examine how unstable the incomes of the individuals have been throughout a given time period, and address questions related to economic insecurity and persistence of poverty (Fields and OK, 1999). ${ }^{10}$

This discussion demonstrates that a crucial preliminary step of any sort of mobility analysis is the clarification of the particular facet of the notion of "income mobility" that one is seeking. But even after one is clear about the particular aspect of mobility that (s)he is interested in, it is, of course, not readily clear which mobility measures are the "right" ones 
for the job; a problem only too familiar from the measurement theories of income inequality and economic poverty.

By far the most common way of specifying a mobility measure is to choose one that, on the face of it, has some intuitively appealing properties. Of course, the result is a multiplicity of measures, and a highly non-unified (and vastly sparse) theory of income mobility measurement. ${ }^{11}$ In this respect, the mobility measurement theory has not yet reached comparability with the theory of inequality measurement, where the axiomatic approach is now dominant. ${ }^{12}$ It is indeed regrettable that axiomatics have not been used more often than they have in the literature on "income mobility".

There are, however, a small number of promising axiomatic approaches to mobility measurement, and in fact, we shall outline one such approach in the following section. Before doing this, however, we should first turn our attention to some other fundamental issues concerning the notion of "income mobility".

\subsection{Relative vs. absolute mobility}

Is mobility a relative or an absolute concept? The following examples may help sharpen one's perceptions on this matter:

$$
\begin{aligned}
& \text { I: } \quad(1,3) \rightarrow(1,3) \\
& \text { IV: }(1,3) \rightarrow(2,6) \\
& \text { V: }(2,6)-(4,12)
\end{aligned}
$$

How do the income mobilities embodied in these distributional transformations compare?

One who feels that at least some income mobility has taken place in process IV but not in process I would not, in effect, be viewing (at least a particular aspect of) income mobility as a strongly relative concept. By definition, a strongly relative (i.e., intertemporally scale invariant) mobility index $f$ must satisfy $f(\lambda x, \alpha y)=f(x, y)$ for all $\lambda, \alpha>0$ and all $x, y \in R_{.}^{n}$ (Shorrocks, 1993). Since the initial income shares are maintained during the transformations I, IV and $\mathrm{V}$, a strongly relative mobility measure would report that the level of mobility is precisely the same in all of these processes, namely, nil. Consequently, any analyst who holds that "process IV is more mobile (in the sense "...") than process I" implicitly rejects intertemporal scale invariance, and is therefore not a strong relativist in that sense. In particular, if one wishes to view income growth as an integral part of the mobility concept that (s)he is interested in, then (s)he would not choose a strongly relative mobility measure. 
The concept of relativism is also used in a weaker sense in the literature. We say that a mobility measure $f$ is (weakly) relative if $f(\lambda x, \lambda y)=f(x, y)$ for all $\lambda>0$ and all $x, y \in R_{*}^{n}$. A relative measure may or may not report positive mobility in the case of transformations IV and V, but it would necessarily record the same level of mobility in these processes.

Mobility measures in common use are generally (weakly) relative; the relativist approach seems to be as dominant in the income mobility literature as it is in the theory of income inequality measurement. ${ }^{13}$ Indeed, the majority of analysts seem to be interested in the variation of the income shares or rank orders of the agents through time, and hence, conduct their analyses by means of strongly relative measures. Yet there is at least one -- "largely ignored" -- sense in which even a relative inequality adherent might not be satisfied with the performance of relative mobility indices which aim to capture the movement aspect of income mobility. While most people feel that the notions of income inequality and income growth are largely independent concepts, it seems reasonable to view the movement aspect of mobility as closely related with income growth. If Robinson Crusoe's income shifts from $\$ 0$ to $\$ 1000$ in a given time interval, few of us would regard his one-person economy as highly immobile (in the sense of lack of movement). On the other hand, a relative mobility measure would assign the minimum level of mobility to this economy; a hardly pleasing result.

Similarly, taking the growth dimension into account, one might justly maintain that there is more mobility in process $\mathrm{V}$ than in process IV, for everyone's income doubled in both cases, transformation $\mathrm{V}$ depicts twice as much income growth. Indeed, some people may choose to declare the process $\mathrm{V}$ as "twice as mobile as" process IV precisely for this reason.

Generalizing this reasoning, therefore, one may argue that a transformation $\lambda x-\lambda y, \lambda>0$ involves exactly $\lambda$ times more movement than $x \rightarrow y$ for there is $\lambda$ times more (per capita) income growth in the former transformation than the latter. In other words, (positive) linear homogeneity may be viewed as a reasonable property at least for mobility indices which target the movement aspect of income mobility.

On the other hand, invariance properties for inequality, poverty and/or mobility indices are, in general, motivated by the degree of coherence they introduce into the measurement exercise. Indeed, one would presumably like a "good" mobility measure to behave in a consistent manner when the variables of measurement go through certain kinds of 
simultaneous changes. Therefore, if we accept the solution of relativity for (at least certain) mobility measures, we would need some other kind of invariance property that would provide a particular consistency check for indices of income movement.

Among the invariance properties in accordance with the movement facet of income mobility is translation invariance which forces the analyst to focus on absolute changes in income levels of the constituent individuals rather than relative ones. Naturally enough, such measures are called absolute, and maintain that the level of mobility associated with a certain transformation would not be altered if the same dollar amount is added to everybody's income in both the initial and final distributions of this transformation. Consequently, among other things, absolute mobility measures allow one to talk about income mobility in terms of total dollars (or per capita dollars), and hence, may well prove useful in policy oriented mobility studies in a way analogous to absolute inequality indices (cf. Blackorby and Donaldson, 1980, p. 107)..$^{14}$

Are there interesting absolute income mobility measures which satisfy linear homogeneity? ${ }^{15}$ We shall argue in section 3 that the answer to this question is yes, and demonstrate that such mobility measures (which may be called compromise mobility indices by analogy to the corresponding measures in the literature on income inequality; see Kolm, 1976 , and Ebert, 1988) possess a number of rather desirable properties. ${ }^{16}$

\subsection{Structural vs. exchange mobility}

As noted by Markandya (1982), pp. 307-8, "within the sociological literature a distinction is made between changes in mobility that can be attributed to the increased availability of positions in higher social classes and those changes that can be attributed to an increased intergenerational movement among social classes, for a given distribution of positions among these classes." (Emphasis in the original). Let us try to clarify these two types of mobility within the context of income mobility again by means of simple examples:

$\mathrm{VI}: \quad(1,2,3) \rightarrow(3,2,1)$

VII: $\quad(1,2,3) \rightarrow(2,1,3)$

VIII: $(1,2,3) \rightarrow(1,2,6)$

IX: $\quad(1,2,3) \rightarrow(2,3,6)$

Notice that the initial (anonymous) income distribution is preserved in processes VI and VII. In this sense, the structure of the economies in VI and VII did not change, and thus the 
observed income mobility in both situations is due entirely to the movement of agents among the available income levels (classes). One may deem that process VI depicts more income mobility than VII solely because of the differences in exchange mobility, for the crosssectional distributions are identical between these two processes. On the other hand, we observe that the structures of the initial income distributions are altered in processes VIII and IX. Moreover, since the income ranks of the individuals are preserved, and since nobody's income is decreased, it may be reasonable to say that there is no exchange mobility in these processes; to the extent that IX is more mobile than VIII, the difference is due entirely to differences in structural mobility.

Attempts have been made in the literature to date to decompose total mobility into exchange mobility and structural mobility components. For example, Markandya (1984) proposed two alternative procedures to do just that: (i) define exchange mobility as the proportion of the change in welfare that could have been obtained if the income distribution stayed constant through time, and let structural mobility be the balance of the total welfare change; (ii) define structural mobility as the change in welfare that would have taken place if there had been no mobility, and (this time) let exchange mobility be defined as the residual. It is not readily clear which procedure yields a more useful decomposition, and one might have to make a choice in this regard, for it is observed empirically that the conclusions of these two disaggregation procedures may be radically different (cf. Markandya, 1984). ${ }^{17}$

All in all, the present literature on income mobility falls short of providing an exact, robust decomposition of total mobility into its basic sources. Nevertheless, there are some recent studies which seem promising in this regard; see, for instance, subsection 3.3 and Silber (1995).

\subsection{Transition matrices}

A considerable portion of the literature on income mobility measurement uses "transition matrices" rather than distributional transformations. Suppose that we have specified $m$ income ranges by one criterion or another. The transition (mobility) matrix induced by a transformation $x \rightarrow y$ is then defined as the matrix $P(x, y)=\left[p_{r s}(x, y)\right] \in R_{+}^{m e m}$, where $p_{r s}(x, y)$ is the proportion of people that were in class $r$ in the distribution $x$ and have now moved to class $s$. By definition, we have $\sum_{s=1}^{m} p_{r s}(x, y)=1$ for all $r$, that is, a transition matrix is necessarily stochastic. 
Transition matrices are particularly useful devices for summarizing the mobility content of distributional transformations. Indeed, they provide a simple picture of the "movement" of the individuals among the specified income classes, and they can thus be quite telling at times. Moreover, as we shall see in section 5, they can be related to stochastic models of income mobility in a natural way, letting one develop easily interpretable mobility measures only in terms of the transition matrices.

Nonetheless, the simplicity provided by transition matrices comes at a cost; summarizing a distributional transformation by a transition matrix usually results in a loss of information. Indeed, one cannot in general recover the transformation that has induced the transition matrix by using only the information provided by the matrix. Moreover, the conclusions based on transition matrices are often robust with respect to the choice of income groups, and in any event, neglect the individual income variations that take place within the specified classes. Therefore, great care should be taken when conducting a transition matrix analysis, and perhaps the analysis must be supplemented by mobility measures that utilize directly the data from the distributional transformations.

It is easy to demonstrate what may go wrong with studying mobility only in terms of transition matrices. Consider a society of 101 people, and suppose that we are interested in two income ranges: "below 5" (low income) and "above 5" (high income). Which one of the following two transformations exhibits more mobility?

$X: \quad(1, \ldots, 1,10) \rightarrow(10, \ldots, 10,10)$

$\mathrm{XI}: \quad(1,10, \ldots, 10) \rightarrow(10,1,10, \ldots, 10)$

Most people would presumably see more mobility in the process $\mathrm{X}$ than in XI due to the much larger number of persons who actually change classes in $\mathrm{X}$. (While 100 people "move up" in X, only one person "moves up" and one person "moves down" in XI.) However, the transition matrix associated with process $X$ is $\left[\begin{array}{ll}1 & 0 \\ 1 & 0\end{array}\right]$, while that induced by process $X I$ is $\left[\begin{array}{cc}0.99 & 0.01 \\ 1 & 0\end{array}\right]$, adopting the convention that the first row and column correspond to the "high income" class. An analyst who is confronted with only these two transition matrices will be tempted to say that process $\mathrm{X}$ is less mobile than $\mathrm{XI}$, for the latter matrix indicates that a larger fraction of the rich actually "moves down" in the process. 
The paradoxical outcome of this particular transition matrix analysis is due to the radically different number of agents in the respective classes of the processes $\mathrm{X}$ and $\mathrm{XI}$. This difficulty, however, could easily be avoided by defining the income classes so that there are always exactly $n / m$ individuals in each group. Such transition matrices are called fractile matrices; a common example is the decile (or quintile) matrices where $m=10(m=5)$ and the $r$ th group corresponds to the $r$ th decile (quintile) of the distribution. ${ }^{18}$ As we shall see in subsection 4.3, fractile matrices are particularly useful for the analysis of mobility that is concerned with the rerankings of individuals through time, and/or with the persistence of relative poverty.

However, mobility analyses based on fractile matrices are not without problems. First, a fractile matrix (like all transition matrices) ignores the income churning that takes place within subgroups. Second, it does not take into account the fact that the absolute income changes that are needed to move between classes would be radically different in the lower and higher fractiles due to the empirically observed positive-skewness of the income distributions. Finally, a fractile matrix may fail to reflect the effect of income growth on the mobility pattern of the society. For instance, the process, $(1,10) \rightarrow(1+\varepsilon, 10+\varepsilon)$ would be viewed as "completely stagnant" for any $\varepsilon>0$ by the fractile matrix of the transformation (with $m=2$ ). Naturally, one may argue that this is a reasonable conclusion since no rank reversal takes place in the process. Yet, one is presumably interested in 'rerankings' for reasons related to intertemporal equality, and the specified process is obviously an equalizing one. (In fact, the second period distribution leads to perfect equality as $\varepsilon \rightarrow \infty$ ). This aspect would have been completely missed, had we evaluated the process only in terms of an associated fractile matrix.

Of course, this does not mean that fractile matrices should not be used in practice. They are indeed very useful devices in assessing the essential "reranking" content of distributional transformations, and they help us answer questions related to low income dynamics and persistence of (relative) poverty. Rather, we maintain that, given the multifarious nature of "income mobility", it might be useful to complement other fractile mobility analyses with other mobility measurement methods. We shall return to this point later in the survey. 


\section{An Axiomatic Approach to Income Movement Measurement}

We have argued above that an important facet of the income mobility concept concerns the movement of incomes through time. But how can one measure movements of incomes? In this section, we shall attempt to answer this question drawing upon the recent axiomatic approaches developed by Fields and Ok (1998) and Mitra and Ok (1998).

\subsection{A class of absolute mobility measures}

As noted in Fields and Ok (1996); asking how much income mobility has taken place in $x \rightarrow y$ may be rephrased as asking how distant $x$ and $y$ have become for an appropriate distance function $d_{n}: R_{*}^{2 n} \rightarrow R_{+} \cdot{ }^{19}$ This basic idea is, in fact, more powerful than it first seems. To see this, suppose that we are interested in absolute income mobility, and thus, wish to view the distance function as measuring the total absolute income movement observed in a given distributional transformation. In view of the discussion presented in subsection 2.3, therefore, two basic assumptions to posit on $d_{n}$ are translation invariance (TI) and linear homogeneity (LH). ${ }^{20}$

We may introduce further structure to the measurement problem by postulating the following fundamental axiom:

Weak Decomposability (WD). For $n \geq 2$

$$
d_{n}(x, y)=G_{n}\left(d_{1}\left(x_{1} y_{1}\right), \ldots, d_{1}\left(x_{n}, y_{n}\right)\right)
$$

for some symmetric, nonzero, strictly increasing and continuous $G_{n}: R_{+}^{n} \rightarrow R_{+}$.

This postulate clarifies the nature of the income mobility concept we are seeking here, namely, that of the aggregate income movement. (This interpretation should be kept in mind throughout this section). One may view WD as a fundamental axiom that any measure of income movement should satisfy. Indeed, this property asserts that total income mobility is a nontrivial and strictly increasing function of the personal mobility levels of the constituents of the society. In other words, $G_{n}$ is basically a function that aggregates the individual income movements. While continuity of this function is a standard regularity assumption, the symmetry of it ensures the impartial treatment of each agent.

Now, it seems plausible that the level of total movement would never decrease upon addition of an individual to the population, and would be unaltered if the new individual 
experienced no income change. A slight strengthening of this idea is that, in the context of populations of different sizes, if equals are added to equals, the results are equal. (Recall that we are seeking a total movement measure at the moment; per capita movement measures will be taken up later). This leads us to the following independence property:

Population Consistency $(P C)$. For $n \geq 3, x, y \in R_{+}^{n-1}, z, w \in R_{+}^{n-2}$ and $a, b \geq 0$ $d_{n-1}(x, y)=d_{n-2}(z, w)$ implies $d_{n}((x, a),(y, b))=d_{n-1}((z, a),(w, b))$.

It is, in fact, possible to obtain a two-parameter characterization of the class of measures induced by these axioms:

Fact 1: (Mitra and OK, 1998) Let the distance functions $\left\{d_{n}: R_{+}^{2 n} \rightarrow R_{+}: n \geq 1\right\}$ satisfy TI, LH, WD, and PC. Then, and only then, there exist $\alpha \geq 1$ and $\gamma>0$ such that

$$
d_{n}(x, y)=\gamma\left(\sum_{i=1}^{n}\left|x_{i}-y_{i}\right|^{\alpha}\right)^{1 / \alpha} \text { for all } x, y \in R_{+}^{n}{ }^{21}
$$

This is a quite useful observation yielding a familiar class of movement measures axiomatically. As long as one's intuition about (total) movement is in accordance with the above axioms, therefore, the "right" mobility index must be of the form of a Euclidean distance. Nevertheless, Fact 1 has limited applicability, for the characterized class still contains uncountably many measures, none of which is "obviously" better than the others. In what follows, we consider two ways of overcoming this problem.

\subsection{An income movement index}

Fields and Ok (1996) propose a separability axiom for income movement measures to the effect that the marginal contribution of one individual's income change to total movement is independent of the other agents' personal mobility levels:

Individualistic Contribution (IC). For any $n \geq 2$ and $x, y, z, w \in R_{+}^{n}$ such that $x_{1}=z_{1}$ and $y_{1}=w_{1}$,

$$
d_{n}(x, y)-d_{n}\left(x,\left(x_{1}, y_{2}, \ldots, y_{n}\right)\right)=d_{n}(z, w)-d_{n}\left(z,\left(z_{1}, w_{2}, \ldots, w_{n}\right)\right)
$$


Although this postulate might be considered less compelling than the previous ones, the implications of it seem worth exploring since such separability axioms are commonly used in the economics of choice and welfare. Indeed, the implications of this strong axiom also turn out to be strong. In our setting, IC, in fact, refines the class of measures obtained in Fact 1 to a singleton:

Fact 2: (Fields and OK, 1996) Let $\left\{d_{n}: n \geq 1\right\}$ satisfy TI, LH, WD, PC and IC, and assume that $d_{1}(1,0)=d_{1}(0,1)=1$ as a normalization. Then, and only then,

$$
d_{n}(x, 1 y)=d_{n}^{0}(x, y) \equiv \sum_{i=1}^{n}\left|x_{i}-y_{i}\right| \text { for all } x, y \in R_{+}^{n} .{ }^{22}
$$

That is, given a natural normalization condition, the unique (total) income mobility measure characterized by the above axioms is the sum of the absolute values of income changes for each individual in the society.

Of course, a total mobility measure may cause problems when the sizes of the groups being analyzed vary. Therefore, in empirical applications, one might rather be interested in per capita and/or percentage mobility measurement. But once $d_{n}^{0}$ is accepted as a satisfactory measure of total movement, we readily obtain per capita and percentage movement indices as

$$
m_{n}^{0}(x, y) \equiv \frac{\sum_{i=1}^{n}\left|x_{i}-y_{i}\right|}{n} \text { and } p_{n}^{0}(x, y) \equiv \frac{\sum_{i=1}^{n}\left|x_{i}-y_{i}\right|}{\sum_{i=1}^{n} x_{i}}
$$

respectively. (The analogy with the notions of total, per capita and percentage, say GNP-growth, is straightforward.) These descriptive indicators are likely to be more useful than $d_{n}^{0}$ in measuring the movement aspect of income mobility in practice. ${ }^{23}$

\subsection{Decomposition of $d_{n}^{0}$}

As noted above, the literature has not until now produced a mobility measure that is decomposable into components akin to "structural mobility" and "exchange mobility" as used by sociologists in thinking about occupational mobility. However, it is not difficult to establish that the mobility indices $d_{n}^{0}, m_{n}^{0}$, and $e_{n}^{0}$ are exactly decomposable into analogous components as follows. 
Let us first define the basic concept of movement due to a transfer of income. This involves transfers of income from some individuals to others, holding total income the same. Take for instance the transfer of $\$ 3$ from individual 3 to individual $1:(1,2,3) \rightarrow(4,2,0)$ Using $d_{n}^{0}$, total mobility is $\$ 6$, because $\$ 3$ is gained by individual 1 and $\$ 3$ is lost by individual 3. More generally, given $x \rightarrow y$ with $\sum_{i=1}^{n} y_{i} \geq \sum_{i=1}^{n} x_{i}$ we may define total movement due to the transfer of income $T(x, y)$ as twice the amount lost by the losers, i.e.,

$$
T(x, y) \equiv 2\left(\sum_{\left\{i: y_{l}<x_{i}\right\}}\left(x_{i}-y_{i}\right)\right)
$$

("Twice" because every dollar lost by a loser is gained by a winner.) ${ }^{24}$ Yet, in a shrinking economy wherein $\sum_{i=1}^{n} y_{i} \leq \sum_{i=1}^{n} x_{i}$ total movement due to the transfer of income, $T(x, y)$ is instead defined as twice the amount won by the winners.

The next concept is movement due to economic growth. When growth occurs, winners can win without anyone losing. Suppose that someone experiences an income gain because of economic growth, e.g., $(1,2,3) \rightarrow(1,2,6)$. Using $d_{n}^{0}$, total mobility is $\$ 3$, all attributable to the gain in income for individual 3. More generally, given $x-y$ with $\sum_{i=1}^{n} y_{i}>\sum_{i=1}^{n} x_{i}$ we define total movement due to economic growth in terms of the usual measure of economic growth: $K(x, y) \equiv \sum_{i=1}^{n} y_{i}-\sum_{i=1}^{n} x_{i}$. Likewise, in a shrinking economy, total movement due to economic contraction is defined as $K^{\prime}(x, y) \equiv \sum_{i=1}^{n} x_{i}-\sum_{i=1}^{n} y_{i}$.

We may now state the following decomposition result:

Fact 3: (Fields and Ok, 1996) Let $n \geq 1$ and $x, y \in R_{+}^{n}$.

(a) If $\sum_{i=1}^{n} y_{i} \geq \sum_{i=1}^{n} x_{i}$, then $d_{n}^{0}(x, y)=T(x, y)+K(x, y)$

(b) If $\sum_{i=1}^{n} y_{i} \leq \sum_{i=1}^{n} x_{i}$, then $d_{n}^{0}(x, y)=T^{\prime}(x, y)+K^{\prime}(x, y)$

The per capita mobility and percentage mobility measures $m_{n}^{0}$ and $p_{n}^{0}$ may be decomposed similarly.

By virtue of these decompositions, we conclude that $d_{n}^{0}, m_{n}^{0}$ and $p_{n}^{0}$ satisfy two important properties. First, they are sensitive to transfers of income in the sense that, for given initial and final income totals, the larger are the absolute values of income changes for the constituent individuals, the more income mobility there is. Second, they are sensitive to 
improved (or diminished) economic opportunities in the aggregate, i.e., given a fixed level of transfer mobility, the larger the change in total income in the cross-sectional income distributions, the more mobility there is. For those who believe that income mobility is a function of both of these components, therefore, these mobility indices present themselves as useful measures of movement-mobility.

\subsection{A partial ordering approach}

In subsection 3.1, we presented a number of appealing axioms for income movement measures and obtained the class of mobility indices entailed by these axioms. Mitra and OK (1998) go on to define the following partial mobility ordering of income distribution transformations: $x \rightarrow y$ is more mobile than $z \rightarrow w$ (written as $(x \rightarrow y) \nabla_{M O}(z \rightarrow w)$ ) if, and only if, $x \rightarrow y$ is declared more mobile than $z \rightarrow w$ by all mobility measures (defined as distance functions) which satisfy TI, LH, WD and PC. In view of Fact 1 , we have

$(x \rightarrow y) \triangleright_{M O}(z \rightarrow w)$ if and only if $\sum_{i=1}^{n}\left|x_{i}-y_{i}\right|^{\alpha} \leq \sum_{i=1}^{n}\left|z_{i}-w_{i}\right|^{\alpha}$ for all $\alpha \geq 1$.

As stressed by Mitra and Ok (1998, p. 95), "the intuitive support of the proposed mobility ordering, is rather similar to that of the Lorenz ordering ... Whenever a transformation is ranked higher by this ordering than the other, there is a clear sense in which one may conclude that the former process is unambiguously more mobile than the latter."

There is, however, an obvious problem with the ordering $\triangleright_{M O}$ : its dependence on the parameter $\boldsymbol{\alpha}$. Indeed, this dependence makes it practically impossible to rank distributional transformations by $\triangleright_{M O}$ except in trivial cases. Nevertheless, there are some easy-to-check sets of sufficient conditions (which do not depend on the parameter $\alpha$ ) for $\nabla_{M O}$ to apply. An illustration follows:

Fact 4: (Mitra and Ok, 1998). For any $x, y, z, w \in R_{+}^{n}, n \geq 1,(x \rightarrow y) \triangleright_{M O}(z \rightarrow w)$ holds if, and only if,

$$
\sum_{i=1}^{k} \Delta_{i}(x, y) \geq \sum_{i=1}^{k} \Delta_{i}(z, w) \text { for } k=1, \ldots, n .
$$

where $\Delta_{i}(x, y)$ is the $i$ th largest element of $\left\{\left|x_{r}-y_{r}\right|: r=1, \ldots, n\right\}$ (and similarly for $\left.\Delta_{i}(z, w)\right)$. 
Consider the transformations $(3,5,9) \rightarrow(13,8,1)$ and $(2,10,4) \rightarrow(11,19,6)$ for example. Can we rank these transformations by $\triangleright_{M O}$ ? (Or better, ask yourself which transformation exhibits more movement than the other?) An easy application of Fact 4 shows that one can indeed: the former transformation is "unambiguousiy" more mobile than the latter.

Mitra and OK (1998) provide several other sets of sufficient conditions which are considerably stronger than those reported in Fact 4. However, while these conditions may prove adequate in enabling one to apply $\triangleright_{M O}$ in some practical cases, they do not reflect the full potential of this descriptive mobility ordering. Obtaining a parameter-free characterization of $\nabla_{M O}$ remains as an open problem at the moment.

\subsection{A final note}

We have found above that the axiomatic method provides one with powerful tools to analyze the income mobility observed in a given distributional transformation. Indeed, as long as one wishes to study the aggregate income movement observed in a given transformation on the basis of individual income changes, the techniques summarized above might well prove useful. Yet it is important to note that this particular measurement theory should not be expected to be suitable for all studies concerning income mobility. For instance, if one is viewing the notion of mobility in terms of "independence of final period income from initial income level," then the above approach is obviously not the right one to follow (recall subsection 2.2).

Similarly, if one's concept of mobility is sensitive to the rerankings of the agents, none of the mobility measures discussed above is appropriate (see section 4.1 for more on this). To see this, notice that $d_{n}^{0}((10,20),(10+\varepsilon, 20-\varepsilon))$ is a continuous function of $\varepsilon$ on $[0,10]$, while one may wish to have a jump discontinuity at $\varepsilon=5$ due to the occurrence of the rank reversal. Indeed, the fractile matrix of the distributional transformation $(10,20) \rightarrow(10+\varepsilon, 20-\varepsilon)$ would be $\left[\begin{array}{ll}1 & 0 \\ 0 & 1\end{array}\right]$ for all $0 \leq \varepsilon<5$, and $\left[\begin{array}{ll}0 & 1 \\ 1 & 0\end{array}\right]$ for all $5<\varepsilon \leq 10$, and this in turn indicates that there is a rank reversal at $\varepsilon=5$. On the other hand, while $d_{n}^{0}$ captures the "movements" that occur in the process for $0 \leq \varepsilon<5$, the associated fractile matrix would fail to do so.

The upshot of this example is that the mobility measurement technique outlined above and the usual approach based on fractile matrices are essentially complementary; movement- 
mobility analysis would be enriched if both methodologies were to be employed simultaneously. ${ }^{25}$

\section{Welfarist Approaches to Income Mobility Measurement}

There is a sizable literature which studies the measurement of income (and social) mobility from a welfarist angle. ${ }^{26}$ In this literature, "mobility is seen in terms of its implications rather than from a direct consideration of what is meant by mobility" (Atkinson, 1981 , p.71). Of course, as Markandya (1982, p. 457), puts it "the presumption here is that a society becomes mobile as a result of the removal of social impediments, which prevent individuals from having an economic role that fully utilizes their natural abilities, and the removal of social privileges, which give them roles that could be better performed by someone else." In this sense, welfarist studies usually view income mobility from the origin independence angle, and in fact, sometimes identify it with the notion of equality of opportunity. In such studies, income mobility is thus valued on its own right (but see Conlisk, 1974, and Atkinson, 1980. $)^{27}$

The first step in devising a welfarist income mobility measure is, of course, to specify a social welfare function (SWF) associating an income distribution stream with a (cardinal) level of aggregate welfare. ${ }^{28}$ Given the social welfare function, a variety of methods can be used to extract a particular mobility measure. In the following subsections, we examine a number of such methods. ${ }^{29}$

\section{4.l King's index}

In an influential study, King (1983) suggests that summary statistics of the rerankings observed in the transformation $x \rightarrow y$ can be interpreted as mobility measures. To outline the basic elements of King's approach, let $z_{i}$ stand for the income level agent $i$ would have obtained if his/her rank order did not alter during the process $x \rightarrow y$, and consider an intertemporal SWF $W: R_{++}^{2 n} \rightarrow R$ defined as $W=W(x, y)=W(y, s)$ where $w: R_{+}^{2 n} \rightarrow R$ is any continuous and strictly increasing function and $s=\left(s_{1}, \ldots, s_{n}\right)$ with $s_{i}=\frac{\left|z_{i}-y_{i}\right|}{\mu(y)}$ for all $i$. ${ }^{30}$ So long as one wishes to measure income mobility only in terms of rank orders of individuals, (s)he must agree that there is no mobility in $x \rightarrow y$ whenever $s=0_{n}$ where $0_{n}=(0, \ldots, 0) \in R^{n}$. Then, however, the index $M(x, y)=1-\frac{1}{\theta(x, y)}$ where 


$$
w\left(\theta(x, y) y, 0_{n}\right)=w(y, s)
$$

measures the proportion of total income which one would be just willing to sacrifice in order to have the degree of mobility (rerankings) we observe in $x \rightarrow y$ rather than having no mobility. ${ }^{31}$ (By the continuity and monotonicity assumptions, $\theta(x, y)$ is well-defined and $\theta(x, y) \geq 1)$.

By postulating strong assumptions on the structure of $w$ therefore, we may obtain specific mobility indices. For instance, if

$$
w(y, s) \equiv\left[\begin{array}{l}
\frac{1}{\eta} \sum_{i=1}^{n}\left(y_{i} e^{\gamma s_{i}}\right)^{\eta}, \eta \neq 0 \\
\sum_{i=1}^{n}\left(\log y_{i}+\gamma s_{i}\right), \eta=0
\end{array}\right.
$$

we have King's mobility index:

$$
M_{k}(x, y) \equiv 1-\frac{1}{\theta(x, y)} \equiv\left[\begin{array}{l}
1-\left[\frac{\sum_{i=1}^{n} y_{i}^{\eta}}{\sum_{i=1}^{n}\left(y_{i} e^{\gamma s}\right)^{\eta}}\right]^{1 / \eta}, \eta \neq 0 \\
1-\exp \left[\frac{-\gamma}{n} \sum_{i=1}^{n} s_{i}\right], \eta=0
\end{array}\right.
$$

Here $\gamma \geq 0$ represents the degree of immobility aversion (see Chakravarty, 1984, for a generalization of this mobility measure).

We stress that King's index (and more generally $1-\frac{1}{\theta(x, y)}$ ) is derived only in terms of the rank order changes in a given transformation $x \rightarrow y$. Therefore, one should be careful in interpreting the conclusions of $M_{K}$ which is likely to yield somewhat extreme results at times. For instance, King's index (and his approach in general) sees the same level of income mobility in the processes $(1,2) \rightarrow(1,2)$ and $(1,2) \rightarrow(1,200)$ namely zero level of mobility; a hardly intuitive conclusion. $M_{K}$, therefore, presents itself as a useful tool only in estimating the welfare value of the aggregate rerankings of a given distributional transformation. 
It is illuminating to compare the behavior of King's index with $d_{n}^{0}$ introduced in subsection 3.2. While $d_{n}^{0}$ ranks the mobility of $(1,2) \rightarrow(1,200)$ higher than $(1,2) \rightarrow(1,2)$ it is by definition insensitive to rerankings of the individuals through time. For instance, while $d_{n}^{0}$ sees the same mobility in the process $(1,2) \rightarrow(1,4)$ as in $(1,2) \rightarrow(3,2)$, King's index ranks the latter transformation "more mobile" than the former in accordance with the intuition that rerankings matter. Unfortunately, the present literature falls short of developing a measurement method which incorporate both the number of dollars moved and the number of rerankings that have occurred in a distributional transformation. This important task remains to be fulfilled in future research.

\subsection{Chakravarty-Dutta-Weymark index}

In their important contribution to ethical measurement of income mobility, Chakravarty, Dutta and Weymark (1985) focus on welfarist measures of strongly relative income mobility, and like Shorrocks (1993), declare the transformation $x \rightarrow y$ as completely immobile if, and only if, the income shares of individuals are maintained through time, i.e., $\frac{x_{i}}{\mu(x)}=\frac{y_{i}}{\mu(y)}$ for all $i$. Consequently, the process $x-\frac{\mu(y)}{\mu(x)} x$ is deemed to exhibit complete (relative) immobility.

Now consider an intertemporal SWF $W: R_{++}^{2 n} \rightarrow R$ such that $W=W(x, y)=w(x+y)$ where $W: R_{+}^{n} \rightarrow R$ is any continuous, strictly increasing and strictly $S$-concave function. By monotonicity and continuity of $W$ there exists a unique $\alpha(x, y)$ which satisfies

$$
W\left(x, \frac{\mu(y)}{\mu(x)} x\right)=w\left(\left(1+\frac{\mu(y)}{\mu(x}\right) x\right)=w\left(\alpha(x, y) 1_{n}\right),
$$

and a unique $\beta(x, y)$ which satisfies

$$
W(x, y)=w(x+y)=w\left(\beta(x, y) 1_{n}\right) .
$$

In this context as others, $\alpha(x, y)$ is the equally distributed equivalent income of the (hypothetical) immobile transformation $x \rightarrow \frac{\mu(y)}{\mu(x)} x$, and $\beta(x, y)$ is the equally distributed equivalent income of $x \rightarrow y$. Clearly, $x \rightarrow y$ exhibits complete (relative) immobility if, and only if, $\alpha(x, y)=\beta(x, y)$ and higher mobility the higher is the ratio $\frac{\beta(x, y)}{\alpha(x, y)}$. Consequently, we may define the following mobility index:

$$
M(x, y) \equiv \varphi\left(\frac{\beta(x, y)}{\alpha(x, y)}\right)
$$


where $\varphi: R_{++} \rightarrow R$ is continuous and strictly increasing. Moreover, by choosing $\varphi(s) \equiv 1-s$, one obtains the Chakravarty-Dutta-Weymark mobility index

$$
M_{C D W}(x, y) \equiv \frac{\beta(x, y)}{\alpha(x, y)}-1
$$

which "measures mobility as the percentage change in the equally distributed equivalent income of the actual aggregate distribution compared with what it would be with the immobile benchmark structure" (Chakravarty et al., 1985, p. 6). ${ }^{32}$

The most striking feature of $M_{C D W}$ is its tendency to distinguish between desirable and undesirable mobility patterns. For instance, it indicates that there is more (ethical) mobility in the transformation $(1,9) \rightarrow(5,5)$ than in $(5,5) \rightarrow(1,9)$. However, the above approach assesses the desirability of the mobility exclusively in terms of income inequality, and ignores, among other things, efficiency matters. For example, $\boldsymbol{M}_{C D W}$ does not see any desirable mobility in $(1,1) \rightarrow(2,2)$ while the final income distribution here is unambiguously better than the initial one. The problem may be solved if we change our focus from relative mobility to absolute (or compromise) mobility, and incorporate the growth aspect of mobility explicitly into the measurement analysis (see subsections 2.4 and 2.5 ). In addition, $M_{C D W}$ is derived via a SWF which depends only on the total income received by the agents through time; there is clearly a need for relaxing this structural assumption and considering broader classes of SWFs. These routes remain to be pursued in future research.

\subsection{Atkinson's income mobility ordering}

In a well-known paper, Atkinson (1981) develops "... 'dominance' conditions under which we can rank mobility processes for a class of social welfare functions satisfying certain general properties. This does not allow a complete ranking; we may be able to rank one process as leading to a higher level of social welfare than some, but not all, other processes" (Atkinson, 1981, p. 71). His approach is best demonstrated by transforming the original data reported in $x \rightarrow y$ into percentile classes and by confining attention to the mobility of individuals among these groupings. Consequently, we assume that a fixed percentage of the population is assigned to each class, that each class is given a certain rank, and that mobility is measured in terms of the number of ranks moved by each person during the transition period. That is, we represent the transformation by the fractile transition matrix 
$P(x, y) \equiv\left[p_{r s}(x, y)\right] \in R_{+}^{m \times m}$ where $p_{r s}(x, y)$ is the proportion of the people that were in class $r$ in the distribution $x$ and have now moved to class $s$ (recall subsection 2.5). In what follows, we shall assume that the $r^{\text {th }}$ fractile of the population is assigned to rank (income status) $r, r=1, \ldots, m$.

A transition matrix $Q=\left[q_{r s}\right] \in R_{+}^{m \times m}$ is said to be obtained from $P \equiv\left[p_{r s}\right] \in R_{+}^{m \times m}$ by a diagonalizing switch if

$$
q_{r s}=p_{r s}+\delta, q_{r, s+1}=p_{r, s+1}-\delta, q_{r+1, s}=p_{r+1, s}-\delta \text { and } q_{r+1, s+1}=p_{r+1, s+1}+\delta
$$

where $\delta>0$; we write $\stackrel{d s}{\rightarrow} Q$ if $Q$ can be obtained from $P$ by finitely many such switches. Since by means of finitely many diagonalizing switches, we can transform any bistochastic transition matrix to the identity matrix (which presumably exhibits the minimum possible level of mobility), there is a sense in which any diagonalizing switch reduces income mobility. In other words, one might argue that $x \rightarrow y$ exhibits more mobility than $z \rightarrow w$ whenever $P(x, y) \stackrel{d s}{\rightarrow} Q(x, y)$. This idea is the premise behind Atkinson's mobility ordering, $\nabla_{A}$, defined as

$$
(x \rightarrow y) \triangleright_{A}(x \rightarrow w) \text { if and only if } P(x, y) \stackrel{d s}{-} Q(x, y) .^{33}
$$

Is there ethical support for Atkinson's ordering? Atkinson (1981) gives an affirmative answer, and shows that $(x \rightarrow y) \triangleright_{A}(z \rightarrow w)$ implies that

$$
\sum_{r=1}^{m} \sum_{s=1}^{m} p_{r s}(x, y) U(r, s) \geq \sum_{r=1}^{m} \sum_{s=1}^{m} p_{r s}(z, w) U(r, s)
$$

for all twice continuously differentiable social evaluation functions $U: R_{+}^{2} \rightarrow R$ such that $\frac{\delta^{2} u}{\delta r \delta s} \leq 0 . .^{34}$ In other words, (utilitarian) social welfare is diminished by a diagonalizing switch if the effect of a switch from state $s$ to $s+1$ in the final period has a stronger impact on the social evaluation function if the switching individual was in state $r$ rather than $r+1$ in the initial period. Consequently, $\nabla_{A}$ appears to have significant ethical support from a utilitarian angle as long as one views the negativity of the cross partials as a reasonable requirement on the social evaluation function.

A point worth noting about $\triangleright_{A}$ is that it does not adhere to the notion of income mobility from the equality of opportunity (or, origin independence) perspective. Indeed, it is easily seen that a distributional transformation represented by the transition matrix $\left[\begin{array}{ll}1 / 4 & 3 / 4 \\ 3 / 4 & 1 / 4\end{array}\right]$ would be ranked more mobile by $>_{A}$ than the transformation represented by the transition matrix 
$\left[\begin{array}{ll}1 / 2 & 1 / 2 \\ 1 / 2 & 1 / 2\end{array}\right]$. It thus seems that Atkinson's notion of income mobility is concerned not with equality of opportunity but rather with income movements (see subsection 5.1 for more on this). Since $\triangleright_{A}$ is primarily an ethical ordering of mobility and since the welfare value of the mobility for the society is usually prescribed in terms of equality of opportunity (or temporal independence), this observation is thought provoking.

Finally, we stress that although Atkinson's ordering is couched in a parallel way to the celebrated Lorenz ordering, the bivariate structure of the mobility measurement problem makes the ethical support of this ordering less apparent. Indeed, in contrast with univariate income inequality measurement theory, the mobility analysis here appears to have a strong utilitarian rooting. Moreover, it is not so clear why it is desirable that the social valuation function have negative cross partials. Of course, one's skepticism about $\triangleright_{A}$ can be traced back to the primitives of Atkinson's measurement theory, the diagonalizing switches. It seems to us that the presumption that any diagonalizing switch decreases mobility is suspect when $p_{r s}$ does not belong to the diagonal of $p(x y)$. After all, it may be argued that if some people move up one class more and an equal number move up one class less, one may want to conclude that mobility is, in fact, unchanged (cf. Fields and Ok, 1996).

Leaving this caveat aside, we conclude by noting that Atkinson's approach is undoubtedly a pioneering one paving way towards a general theory of ordinal measurement of income mobility.

\subsection{Mobility measures based on social utility variations}

In section 3 we considered a number of mobility measures which are defined as certain additively separable aggregations of individual income changes. As pointed out in footnote 16 , one shortcoming of such mobility measures is that they tend to view the distributional transformations $(2,1000) \rightarrow(2,1001)$ and $(2,1000) \rightarrow(3,1000)$ as equally mobile. Indeed, for those analysts who wish to rank the mobilities of these transformations on the basis of changes in individual welfare levels, the law of diminishing returns would provide a justification for the argument that the latter process is more mobile than the former. This suggests defining mobility indices of the form

$$
f_{n}^{U}(x, y) \equiv \frac{1}{n} \sum_{i=1}^{n}\left|U\left(y_{i}\right)-U\left(x_{i}\right)\right| \text { for all } x, y \in R_{++}^{n}
$$


where $U: R_{++}^{n} \rightarrow R$ is a strictly increasing and concave function. Once again $U$ is interpreted here as a social evaluation (utility) function of a social planner (or as the utility function of the representative agent) as is usual in welfare economics. Consequently, $f_{n}^{U}(x, y)$ is basically thought of as the per capita aggregate change in the individual social utility levels experienced during the process $x \rightarrow y$ according to the utilitarian social welfare functional. ${ }^{35}$

Clearly, if $U$ is strictly concave, $f_{n}^{U}$ declares $(2,1000)-(3,1000)$ as strictly more mobile than $(2,1000) \rightarrow(2,1001)$ in accordance with the above mentioned intuition. By the same token, such measures (with a strictly concave $U$ defined over the set of income ranks $\{1, \ldots, m\})$ also satisfy Atkinson's diagonalizing switch axiom discussed above.

It is worth mentioning that any $f_{n}^{U}$ can be decomposed into growth and transfer components (defined in terms of utility) in precisely the way we have described in subsection 3.3. Moreover, any $f_{n}^{U}$ enjoys an additional separability property which may prove quite useful in applications: subgroup decomposability. Indeed, for any partitioning of the society, $f_{n}^{U}$ computes the overall mobility as a weighted average of the subgroup mobility levels where weights are chosen to be the population shares of the subgroups. ${ }^{36}$ Just as in the theory of poverty measurement (cf., Foster, Greer and Thorbecke, 1984), the basic appeal of subgroup decomposability lies in its promise for empirical applications, for it lets one determine exactly the contribution of any population subgroup (which may be defined according to the ethnic, occupational and/or geographic origin) to the overall mobility of the society (see Fields and Ok, 1999, for a further discussion of the issue).

Now, given the definition of $f_{n}$, we may obtain a particularly interesting class of mobility measures by confining our attention to social evaluation functions with constant relative risk aversion (as in the seminal work of Atkinson, 1970). As is well known, the members of this class are necessarily of the form

$$
U^{\sigma}(a)=\left\{\begin{array}{ll}
\frac{a^{1-\sigma}}{1-\sigma} & 0 \leq \sigma \neq 1 \\
\log a, & \sigma=1
\end{array} \quad \text { for all } a>0\right.
$$

Let us denote the class of all mobility indices induced by these utility functions in the way prescribed above by $\Omega$. 
An immediate observation is that $f_{n}^{U^{\circ}}=m_{n}^{0}$, that is, the only absolute mobility measure in $\Omega$ is the per capita (total) mobility index we have (independently) characterized in subsection 3.2. On the other hand, the only relative member of $\Omega$ is the per capita aggregate change of log incomes, that is, the index defined as

$$
f_{n}^{U^{1}}(x, y)=\frac{1}{n} \sum_{i=1}^{n}\left|\log \frac{y_{i}}{x_{i}}\right| \quad \text { for all } x, y \in R_{++}^{n} \cdot{ }^{37}
$$

This is an interesting movement-mobility measure which might be an appealing alternative to $m_{n}^{0}$. We refer the reader to Fields and Ok (1999) for a detailed investigation of the desirable properties of $f_{n}^{U^{1}}$, and an axiomatic characterization of this particular measure which provides a formal justification for concentrating on log-incomes in mobility analyses.

In conclusion, we stress that all of the measures considered in this subsection are measures of mobility which seek to measure the aggregate income variation observed in a given distributional transformation. Therefore, their normative significance is not readily apparent (see footnote 10), and they do not attach a particular weight to rerankings of individuals beyond what is implied by the associated social utility changes. Their conclusions must therefore be supplemented by those of other mobility measures. One method of doing this is to use directionalized versions of $f_{n}^{U^{1}}$; this issue too is discussed formally in Fields and Ok (1999).

\section{The Markovian Approach to Mobility Measurement}

\subsection{Temporal independence vs. aggregate movement}

By its very nature, income mobility is primarily concerned with the time path of the income distribution among identified individuals in a given society. It is therefore not surprising that much of the literature on income mobility measurement utilizes stochastic processes in modeling the generation of such time paths. The most popular example is the simple Markov chain model of mobility where the transitions across agents are independent. The essential primitive of this model is the transition (mobility) matrix of the chain, $P=\left[p_{i j}\right] \in R_{+}^{\text {mxm }}$ where $p_{i j}$ stands for the probability of moving to income state $j$ from income state $i$ within a unit interval of time. (Of course, $\Sigma_{j=1}^{m} p_{i j}=1$ and $m$ is the number of income states.) ${ }^{38}$ When one 
thinks of $P$ as specifying an intertemporal income distribution sequence, for example, $p_{i j}$ may stand for the probability that a parent in income state $i$ will have an offspring in state $j$. (Given this interpretation, therefore, we may interpret the probability vector $\left(p_{i l}, \cdots, p_{i m}\right)$ as the lottery that an agent with a parent from state $i$ faces.) In an intragenerational setting, on the other hand, one usually views the generated income streams as individuals' incomes in successive periods, or as lifetime earnings.

Shorrocks (1978) provides a careful examination of income mobility measures which are defined as continuous functions of the form $M: P \rightarrow R .{ }^{39}$ He suggests the following property for such measures: $M(I) \leq M(P) \leq M(Q)$ where $I$ is the mxm identity matrix and $Q$ is any mxm transition matrix all rows of which are identical. Therefore, the identity matrix is assigned the least level of mobility, for such a mobility matrix generates no transitions between income states. On the contrary, a mobility matrix with identical rows is assigned the largest level of mobility since such a matrix induces perfect temporal (origin) independence (Prais, 1955).

It must be clear at this point that this latter property is only desirable from a temporal dependence point of view; for instance, when assessing the degree to which an offspring's income state is determined by that of the parent in an intergenerational framework. If, on the other hand, one chooses to approach the notion of income mobility from the perspective of total movement rather than that of temporal independence, the axiom is clearly no longer suitable (recall subsection 2.2). For example, while $\left[\begin{array}{ll}1 / 2 & 1 / 2 \\ 1 / 2 & 1 / 2\end{array}\right]$ is a transition matrix which exhibits unambiguously less movement than the matrix $\left[\begin{array}{ll}0 & 1 \\ 1 & 0\end{array}\right]$ Shorrocks' postulate requires that the former matrix be more mobile than the latter one (cf. Bartholomew, 1982 and Kanbur and Stiglitz, 1986). Indeed, this example illustrates that the monotonicity postulate of Shorrocks (1978), which requires that, for all transition matrices $P$ and $Q, M(P)>M(Q)$ whenever $p_{i j} \geq q_{i j}$ for all $i \neq j$ with at least one of the inequalities holding strictly, is incompatible with the above property. This observation teaches us once more that one has to be rather careful in specifying the precise nature of the mobility notion that is targeted in the measurement exercise. ${ }^{40}$ 


\subsection{Monotone mobility matrices}

It has recently been pointed out by several authors that the class of all mobility matrices is simply too large to work with, and there is, in fact, a useful way of refining this class. To introduce this refinement, let us adopt the convention that income state $i$ is represented by income level $x_{i}$ and the income states are ordered from lowest income to highest. Illustrating in an intragenerational context, it seems natural to postulate that an agent starting in income state $i$ is better off than starting in state $i+1$. Yet $x_{i} \leq x_{i+1}$ does not guarantee this; a (regular) transition matrix may well generate a higher lifetime expected income for an agent starting at a lower state. However, we may rule out this possibility by requiring that row $i+1$ of the transition matrix stochastically dominate row $i$. (In an intragenerational framework, for instance, this amounts to the sensible requirement that an agent faces a better lottery in state $i+1$ than $i$ ). This observation leads one to confine attention to monotone mobility matrices first introduced by Keilson and Ketser (1977).

A transition matrix $p$ is said to be monotone if, for all $i=1, \ldots, m-1$,

$$
\sum_{j=1}^{k} p_{i+1, j} \geq \sum_{j=1}^{k} p_{i j} \quad k=1, \ldots, m-1 .
$$

As noted by Conlisk (1990), monotonicity appears to be a compelling property to posit on mobility matrices; it is intuitively appealing and is empirically verified by many studies (cf. Adelman, 1958, Shorrocks, 1976, Bartholomew, 1982 and Dardanoni and Forcina, 1993). Moreover, confining attention to monotone (and regular) transition matrices provides one with considerable analytic structure that leads to many interesting results concerning income mobility measurement. We present here two examples.

Example 1: (Dardanoni, 1995) Consider two Markovian transition matrices $P$ and $Q$ such that the "expected" income distribution at time $t-1$ under the process generated by $P$ is more equal (in the Lorenz sense) than that generated by $Q .{ }^{41}$ A natural question is this: under what circumstances will the "expected" income distribution at time $t$ under the process induced by $P$ remain more equal than that induced by $Q .{ }^{42}$ The answer to this question is quite complicated in general. But if we assume that $P$ is monotone, then one obtains a definitive answer very easily: the income distribution generated by $P$ at time $t$ will remain more equal than that generated by $Q$ if, and only if, $P$ stochastically dominates $Q$; that is,

$$
\sum_{j=1}^{k} p_{i j} \leq \sum_{j=1}^{k} q_{i j} \text { for all } k=1, \ldots, m-1, \quad i=1, \ldots, m
$$


(Dardanoni, 1995, Proposition 1). ${ }^{43}$ In other words, this result "tells us that when the transition matrix of the society with a dominating income distribution is monotone, a necessary and sufficient condition, for the preservation of the dominance relation in the following snapshot is stochastic dominance of the transition matrix" (Dardanoni, 1995, p. 185).

Example 2: (Dardanoni, 1993) Consider an intragenerational Markovian model of income mobility with the transition matrix $P$. Let $X_{i}(P ; \delta)$ denote the (normalized) expected discounted lifetime income of an infinitely lived agent where $\delta \in[0,1]$ is the discount factor; that is, let $X_{i}(P ; \delta)$ be the ith element of

$$
X(P: \delta) \equiv(1-\delta) \sum_{t=0}^{\infty} \delta^{t}\left(x P^{t}\right)=x\left((1-\delta)[I-\delta P]^{-1}\right) \equiv x P(\delta) .
$$

By regularity of $P$ there exists a unique (strictly positive) equilibrium probability vector $\pi$ (which, evidently, identifies the steady-state income distribution). We may thus consider a Bergson-Samuelson type SWF that aggregates the expected discounted lifetime incomes of the agents as a weighted sum:

$$
W: X(P ; \delta) \rightarrow \sum_{i=1}^{m} \pi_{i} \lambda_{i} X_{i}(P ; \delta)
$$

Of course, if we assume that $P$ is monotone, then starting at a lower income state is necessarily a disadvantage so that a natural faimess argument demands that we assume $\lambda_{1} \geq . . \geq \lambda_{m} \geq 0$. Then, however, given the discount factor $\delta$ we can unambiguously rank two regular monotone transition matrices $P$ and $Q$ with the same equilibrium probability vector $\Pi$ as follows:

$$
P \triangleright_{D} Q \quad \text { if and only if } \sum_{i=1}^{m} \pi_{i} \lambda_{i} X_{i}(P: \delta) \geq \sum_{i=1}^{m} \pi_{i} \lambda_{i} X_{i}(Q ; \delta)
$$

for all $\lambda_{1} \geq \ldots \geq \lambda_{m} \geq 0$ and all $x_{i} \leq \ldots \leq x_{m} \cdot{ }^{44}$ (Notice that without the monotonicity assumption, $\triangleright_{D}$ is not necessarily a sensible ordering, for then the condition $\lambda_{1} \geq \ldots \geq \lambda_{m} \geq 0$ cannot be justified on the basis of faimess.) The binary relation $>_{D}$, which we refer to as Dardanoni's ordering, is a particularly interesting mobility ordering since, as noted in Dardanoni (1993, p. 379), it "may be considered (as) the infinite horizon extension to Atkinson's ordering of bistochastic transition matrices for a two period society" which was analyzed in subsection 4.3 . 
Before concluding this example, we note that $\triangleright_{D}$ is, in fact, very easy to check for a given $\delta$, because one can show that, for any monotone and regular $P$ and $Q$ with the same equilibrium vector, we have

$$
P \triangleright_{D} Q \quad \text { if and only if } \quad T^{\prime} \Pi P(\delta) T \leq T^{\prime} \Pi Q(\delta) T
$$

where $T$ is the mxm upper triangular matrix with zeros below the main diagonal and ones elsewhere, and $I$ is the diagonal matrix with the equilibrium probability vector $\pi$ on the diagonal..$^{45}$

These examples illustrate that considerable progress can be made theoretically by concentrating only on monotone Markov chains (identified by monotone transition matrices) in modeling the time paths of distributions of income. Nevertheless, the problem of income mobility measurement can hardly be considered as "solved" upon introduction of monotone mobility matrices; there are several tasks left for future work. First of all, Dardanoni's ordering can rank only mobility matrices with the same steady-state income distribution, which is unlikely to arise in practice if the matrices summarize movements among income classes. Therefore, it will be useful to find a way to extend $\nabla_{D}$ in a way to dispense with this requirement but such an extension will introduce considerations of structural mobility into the analysis, and we are thus led to the problems of decomposition. ${ }^{46}$ It would be quite interesting to see if monotonicity of the mobility matrices will prove useful in this regard. Finally, one must eventually search for the ethical support of Dardanoni's ordering outside the span of Bergson-Samuelson type social welfare functions.

\subsection{A caveat}

The standard Markovian model of income mobility is not without problems. Among others, it is based on two assumptions the validity of which is neither empirically nor theoretically evident: (1) the transition probabilities are constant over time (i.e., the Markov chain is stationary); (2) the probability of going from one state to the other is independent of past history (i.e., the Markov chain is of first-order). ${ }^{47}$ The first assumption is troubling since it may result in a considerable degree of information loss with respect to the inherent mobility exhibited in a given time interval. One can, of course, relax this assumption and rather focus on time dependent Markov chains, but this leads to quite a complicated stochastic process where the tractability of the standard model is unrecoverably lost. The 
second assumption, on the other hand, is refuted by a number of empirical studies (cf. Shorrocks, 1976, Atoda and Tachibanaki, 1980, and Atkinson et al., 1992). ${ }^{48}$

We are therefore at an uneasy crossroads. The first order stationary (and monotone) Markov chain model provides an analytically tractable framework for a fruitful analysis of income mobility, at least theoretically. But one pays a high price for the "nice" structure this model provides, for the two strong assumptions noted above may not hold empirically. In the final analysis, the decision regarding whether or not a Markovian approach should be adopted for a particular income mobility analysis, appears to be a subjective and context-dependent question.

\section{A Final Synopsis}

The income mobility literature is still distressingly far from being unified on how to measure mobility and make mobility comparisons. In part, this is presumably because of lack of agreement on what the underlying concept is, but this is also due to the wide gap (with a few notable exceptions) between those who devise measures of income mobility and those who measure mobility empirically.

One must recall that the inequality literature made genuine progress when empirical and theoretical researchers combined their forces, and there emerged powerful methods of measuring income inequality commanding almost unanimous approval. Moreover, the related literature was able to provide a unified framework within which competing techniques could be studied, and eventually it became clear where the differences lay and what the answers depended upon.

It appears that mobility analysts can beneficially follow the lead of inequality analysts by asking similar kinds of questions:

(1) What do I mean by income mobility? What particular aspects of it am I interested in measuring? What is the normative significance of these aspects?

(2) What sort of a measurement methodology should I use to make ordinal mobility comparisons?

(3) Given my answer to (1), how, axiomatically or otherwise, do I justify the choice of my measurement methodology? Is there an aspect of mobility that this technique misses? What are the remedies? 
As this survey has attempted to clarify, the notion of mobility is truly multi-dimensional, and the social welfare importance of each of these dimensions varies. Even when one is absolutely clear about what aspect of mobility s/he is interested in measuring, we lack measurement techniques which are as widely accepted (and axiomatically justified) as those in the analysis of inequality and poverty. Moreover, the connections between the numerous mobility measurement methods advanced in the literature are unclear. At this stage, therefore, we must conclude that, although much progress has been made in clarifying the meaning and measurement of income mobility especially in the last two decades, much more remains to be done in future research.

\section{Notes}

1. The authors wish to thank Jacques Silber for his patience and encouragement, and Roland Benabou, François Bourguignon, Valentino Dardanoni, James Foster, Stephen Jenkins, Tapan Mitra, Anthony Shorrocks and Ed Wolff for illuminating discussions. The financial support of the Bronfenbrenner Life Course Center at Comell University and the C.V. Starr Center for Applied Economics at New York University are also gratefully acknowledged.

2. There are a number of well-known surveys of this literature; see, for instance, Sen (1973), Eichhorn and Gehrig (1982), Foster (1985) and Lambert (1993).

3. For example, Karcher, Moyes and Trannoy (1995) note that "... since the individuals" positions on the cardinal income scale rarely remain unchanged over time, an increase in a snapshot measure of inequality is clearly consistent with there having been a significant amount of equalizing mobility over time. Only if all individuals' earnings remain constant from period to period will a measure of inequality or welfare give the same result irrespective of the length of the accounting period." This argument is, in fact, at the heart of the welfare policy formulations of many economists; see, inter alia, Friedman (1962), and Becker and Tomes $(1979,1986)$.

4. Throughout this paper, we take "income" as the measure of economic status of interest and "individuals" as the interesting unit of observation. Of course, our discussion apply equally to any real-valued measure of socioeconomic position (e.g., earnings, indices of occupational status, etc.) among any well-defined recipient unit (e.g., households, workers, generations, adult equivalents, etc.).

5. Among the important omissions are the relation between income mobility and the time period of analyses (cf. Shorrocks, 1978a, Creedy, 1985, pp. 97-118, and Creedy, 1992, and references cited therein), and econometric mobility measurement techniques like Galtonian regression towards the mean (cf. Creedy, 1985, Chapter 4, Abowd and Card, 1989, Goldberger, 1989, Zimmerman, 1992, and Atkinson, Bourguignon and Morrison, 1992, pp. 8-14, Moffitt and Gottschalk, 1995, and Maasoumi, 1996, inter alia).

6. See, for instance, Shorrocks (1978a), Maasoumi and Zandvakili (1986), Chakravarty, Dutta and Weymark (1985) and Creedy (1992). Space limitations preclude giving these contributions the attention they deserve.

7. More generally, a correlation-based mobility measure can be defined as $f(x, y) \equiv H(r(\psi(x), \psi(y)))$ where $H:[-1,1]-R$ and $\psi: R_{+}-R$ are any continuous functions which are decreasing and increasing, respectively. (Convention: $\psi(x) \equiv\left(\psi\left(x_{1}\right), \ldots, \psi\left(x_{n}\right)\right.$ ) and similarly for $\psi(y)$.) An example of a correlation-based mobility measure is Har's index, $f_{H}(x, y) \equiv 1-r(\log x, \log y)$, an extensive discussion of which can be found in Shorrocks (1993). 
8. One should not infer from this discussion that movements in the aggregate and origin independence are the only aspects of the "income mobility" concept. One might indeed choose to distinguish between mobility concepts on the basis of aggregate rerankings or intertemporal income inequality (see subsections 4.1 and 4.2).

9. One should, however, be careful in relating the notion of aggregate movements to that of intertemporal equality, for the correspondence is by no means perfect. Indeed, while the transformation $(1,3)-(30,1)$ involves far more movement than process II, there is a clear sense in which this transformation is intertemporally disequalizing as compared with process II. Nevertheless, one might justifiably argue that such examples are unlikely to be encountered in practice, and hence, the movement aspect of income mobility might still be regarded as a telling proxy for intertemporal income equality.

10. Attaching a precise normative significance to "income mobility" is difficult because of the multidimensionality of this concept (see, however, section 4). Indeed, as stated by Jarvis and Jenkins (1996, p. 1), "on the one hand mobility is an indicator of how open society is and the degree of equality of opportunity, and hence a Good Thing. To some, greater inequality may be more tolerable if accompanied by significant mobility. On the other hand, mobility may also be a synonym for income fluctuations and hence economic insecurity, a Bad Thing. Whether income flux is more concentrated amongst the poorest or the richest is likely to influence the overall social verdict."

11. Examples of mobility (or immobility) measures are numerous: the correlation coefficient (McCall, 1973), the rank correlation (Schiller, 1977), the quantile (decile) immobility ratio (i.e., the fraction of people changing no more than one income quantile (decile); Lillard and Willis, 1978, and Gottschalk, 1982), income immobility ratio (i.e., the fraction, of people changing no more than one relative income range; Thatcher, 1971), Hart's index (Hart, 1981), average jump in income rank (Scott and Litchfield, 1994), Shorrocks' index and the Maasoumi-Zandvakili index (Shorrocks, 1978a, 1993, and Maasoumi and Zandvakili, 1986).

12. See, for instance, Chakravarty's contribution in this volume.

13. Even in the case of income inequality, however, there are persuasive arguments that can be made for absolute inequality measures; see Kolm, 1976, and Blackorby and Donaldson, 1980, inter alia.

14. Whether one's perception of mobility is relative or absolute, is, in the final analysis, a subjective issue. In either case, however, it should be noted that one can utilize absolute and relative measures in a complementary way. Indeed, in discussions concerning income growth, for instance, it is standard to make use of the concepts of per capita growth (which is an absolute notion) and percentage growth (which is relative) in a complementary fashion.

15. Unlike inequality measures, mobility measures can be both absolute and relative.

16. One argument that may be made against compromise mobility measures is that they may report the same level of mobility in the processes $(1,100)-(1,101)$ and $(1,100)-(2,100)$. Indeed, assuming that money is valued by a common strictly concave utility function, one might want to argue that the former process is less mobile than the latter in the sense that there is less welfare movement in the former transformation relative to the latter one (see subsection 4.4). We would like to stress, however, that this is not an argument favoring "relativity" over "compromization". Indeed, while the mobility measure (on $R_{t+}^{2 n}$ $g(x, y) \equiv f(x, y) / \sum_{t=1}^{n} x_{i}$ is relative for any compromise $f$, it ranks the above processes in exactly the same way with $f$. Moreover, the first process would be declared less mobile by all mobility measures (compromise or relative) which satisfy a very weak decomposability property (Axiom WD in subsection 3.1), provided that these measures are applied to personal percentage changes of income. (That is, given such an $f$, the relative mobility measure $f_{R}$ defined (on $R_{*}^{2 n}$ as $f_{R}(x, y)=f\left(1_{n}, y / x\right)$, where $1_{n}$ is thendimensional vector of $1 \mathrm{~s}$ and $y / x \equiv\left(y_{1} / x_{1}, \ldots, y_{n} / x_{n}\right)$, would have the property that $\left.f_{R}((1,100),(1,101))<f_{R}((1,100),(2,100))\right)$. 
17. Furthermore, these decompositions depend crucially on the chosen social welfare function and some strong assumptions about the stochastic process that is assumed to generate the mobility of the society. We shall have more to say on these issues in sections 4 and 5 .

18. Since each class is assumed to have exactly the same number of members, the transition matrix $P(x, y) \in R_{+}^{m+n}$ is bistochestic; that is, $\sum_{r-1}^{m} p_{r}(x, y)=\sum_{s=1}^{m} p_{r s}(x, y)=1$. However, it must be noted that the bistochasticity of the transition matrix "does not imply that the distribution is unchanging over time, and that analyses based on quantile transition matrices may confound exchange and structural mobiiity." (Atkinson et al., 1992, p. 15).

19. Dagum (1980), Shorrocks (1982), Ebert (1984) and Chakravarty and Dutta (1987) have used distance functions in the context of inequality measurement. (For an empirical application of such "economic" distance functions, see Silber and Berrebi, 1988). In the context of mobility measurement, Cowell (1985) appears to be the only study where a distance function theoretic approach is pursued. Cowell's development, however, builds upon the concept of a directed distance function, and this distinguishes his work from the standard "economic" distance function approach.

20. That is, we assume that $d_{n}(x, y)=d_{n}\left(x+\alpha 1_{n}, y+\alpha 1_{n}\right)$ and $\lambda d_{n}(x, y)=d_{n}(\lambda x, \lambda y)$ for all $\lambda>0$ and $\alpha$ such that $x+\alpha 1_{n}, y+\alpha 1_{n} \in R_{+}^{n}$, where $1_{n}=(1, \ldots, 1) \in R^{n}$. We note that the analogues of these assumptions are routinely used in the literature in income inequality measurement; see, for instance, Blackorby and Donaldson $(1978,1980)$, Ebert $(1984,1988)$, and Chakravarty and Dutta (1987).

21. See Fields and Ok (1996) for a detailed discussion of the axioms WD and PC, and a related characterization theorem.

22. Note that the metric axioms are not used in this characterization theorem, while they were necessary in Fact 1. This is an appealing feature of Fact 2 , since it is not apparent why an economic distance function should, in fact, satisfy the triangle inequality as an axiom.

23. Notice that $p_{n}^{0}$ is a relative mobility measure, and hence, might appeal to relative mobility adherents. As noted earlier, in our opinion $d_{n}^{0}$ and $p_{n}^{0}$ should be used in a way to complement each other. For example, in the comparison of the transformations IV: $(1,3)-(2,6)$ and V: $(2,6)-(4,12), d_{2}^{0}$ (and, of course, $m_{2}^{0}$ ) report that $\mathrm{V}$ is more mobile than IV since it exhibits a higher level of (per capita) income growth than IV, while $p_{2}^{0}$ notes that the mobilities of these transformations are (percentage-wise) equal since they both have the same percentage income growth.

24. A "winner" here is defined as an agent whose income increases, and a "loser" as a person whose income decreases.

25. This is, in effect, analogous to using the head count and the poverty gap measures together to get a more complete picture of the extent of poverty (this being one of the basic messages of the seminal work of Sen, 1976). Recall that the head count ratio is insensitive to income movements within the poor class while being discontinuous at the poverty line. One the other hand, the poverty gap index is a continuous measure which is not particularly sensitive to income variations that carry individuals above the poverty line. Both of these measures target a different aspect of the general notion of "economic poverty".

26. See, among others, Atkinson (1981), Markandya (1982, 1984), King (1983), Chakravarty (1984), Chakravarty, Dutta and Weymark (1985), Kanbur and Stiglitz (1986), Slesnick (1986), Kanbur and Stromberg (1988), Atkinson, Bourguignon and Morrison (1992), Dardanoni (1991, 1993, 1995) and Gottschalk and Spolaore (1998).

27. Friedman (1962), for instance, takes this argument to the extreme and suggests the substitution of income mobility for income equality as a normative justification of competitive free enterprise capitalism. His classic quotation reads as follows: "Consider two societies that have the same distribution of annual income. In one there is great mobility and change so that the position of particular families in the income hierarchy varies widely from year to year. In the other, there is great rigidity so that each family stays in the same 
position year after year. The one kind of inequality is a sign of dynamic change, social mobility, equality of opportunity; the other, of a status society. The confusion of these two kinds of inequality is particularly important precisely because competitive free-enterprise capitalism tends to substitute the one for the other...capitalism undermines status and induces social mobility" (Friedman (1962), p. 171). Of course, this proposition is hardly self-evident. (Solon, 1992, and Zimmerman, 1992, for instance, provide convincing evidence to the effect that there is a rather high (in the order of 0.4 ) intergenerational correlation in long-run income in the United States. Similar results are reported for Britain as well by Atkinson, 1981b.) Moreover, the nature of the relation between the inequality of snapshot (or lifetime) incomes and income mobility is by no means clear either empirically or theoretically. (Indeed, some empirical studies have found only a weak relationship between income mobility and inequality through time; see Kearl and Pope, 1984.) For an illuminating account of this issue, we refer the reader to Atkinson, Bourguignon and Morrison (1992), pp. 23-29, and references cited therein. See also Shorrocks (1978a), Kanbur and Stiglitz (1986), Kanbur and Stromberg (1988), Conlisk (1989), Basmann, Hayes and Slottje (1991), and Dardanoni (1991, 1995) for related measurement-theoretic examinations.

28. The arbitrariness of the choice of the social welfare function may, in fact, be thought of as the major shortcoming of the welfarist approach (see, however, subsection 4.3). Moreover, by definition of social welfare functions, this approach brings with it all the well-known difficulties associated with the concept of "welfarism" (see Sen, 1979).

29. The following subsections are only meant to provide the reader with some examples of welfarist income mobility measurement techniques, and are by no means comprehensive. See, in particular, the important works of Shorrocks (1978a), Maasoumi and Zandvakili (1986, 1990) and Markandya (1982a).

30. By convention, we shall define a generic SWF on feasible incomes, rather than on utility profiles, in what follows.

31. The basic premise of this approach is analogous to the pioneering work of Kolm (1969) and Atkinson (1970) on income inequality measurement. See also Markandya (1982a) for a similar approach.

32. Chakravarty et al. (1985) provides a useful characterization of $\boldsymbol{M}_{C D W}$ which amounts to determining necessary and sufficient conditions on the functional structure of a mobility index such that there exists a well-behaved $W: R_{+}^{\prime \prime} \rightarrow R$ which would induce the index via the procedure described above. The approach parallels the work of Blackorby and Donaldson (1978), the focus of which was the welfarist measurement of relative income inequality along the lines outlined in Kolm (1969), Atkinson (1970) and Sen (1973). For more on this, see the contribution of Blackorby, Bossert and Donaldson in this volume.

33. While it is not readily clear when a transformation can actually be obtained from another by a finite sequence of diagonalizing switches, Atkinson (1981) and Atkinson et al. (1992) give a characterization of $D_{A}$ which overcomes this difficulty:

$$
(x-y) \nabla_{A}(z-w) \text { if and only if } P_{C U M}(x, y) \leq P_{C U M}(z, w)
$$

where $P_{C U M}(x, y)=\left[\Sigma_{i=1}^{r} \hat{\Sigma}_{j=1}^{s} p_{i j}(x, y)\right] \in R_{+}^{m o m}$ and $P_{C U M}(z, w)$ is defined similarly.

34. See Atkinson and Bourguignon (1982) for various extensions of this result.

35. An analogous idea was developed by Watts (1968) in the context of poverty measurement where it was suggested that one should represent the deprivation of a poor individual in terms of the utility shortfall from the level of utility that would have been enjoyed with an income precisely at the poverty line; see also Zheng (1993), and Foster and Jin (1994).

36. Formally speaking, a mobility measure $f$ is said to be subgroup decomposable, if, for any $x^{j}, y^{j} \in R_{++}^{z_{j}}, j=1, \ldots, J$, such that $\sum_{j=1}^{J} n_{j}=n$,

$$
f_{n}\left(\left(x^{1}, \ldots, x^{J}\right),\left(y^{1}, \ldots, y^{J}\right)\right)=\sum_{j=1}^{J}\left(\frac{n_{j}}{n}\right) f_{n_{j}}\left(x^{j}, y^{j}\right) .
$$


37. Since $f_{\mathrm{n}}^{V^{1}}$ and $f_{\mathrm{n}}^{U^{1}}$ are the only absolute and relative measures in $\Omega$, we should note that the subclass $\Omega_{0} \equiv\left\{f_{n}^{U_{o}}: 0<\sigma<1\right\}$ may be viewed as a one-parameter class of "intermediate" measures of income mobility.

38. Some studies are actually conducted in terms of continuous time Markov chains; see, for instance, Coleman (1964), Bartholomew (1982), Geweke, Marshall and Zarkin (1986) and Kanbur and Stromberg (1988).

39. Among the examples of mobility measures of this form are the trace (Prais, 1995 and Bibby, 1975), and the second largest eigenvalue modulus (Theil, 1972, Shorrocks, 1978, and Sommers and Conlisk, 1979). Provided that the transition matrices under consideration are regular (i.e., $P^{k} \in R_{++}^{k x n}$ for large enough $k$ ), one can define more sophisticated mobility measures like the mean first passage time (Conlisk, 1985, 1990) and weighted trace (Bartholomew, 1982). For formal examinations of these measures and further examples we refer the reader to Bibby (1975), Bartholomew (1982) and Conlisk (1990).

40. Having said this, however, we should note that Shorrocks (1978, pp. 1016-7) argues that "... as more movement is observed it would be normal to expect the class occupied in the future to become less dependent on the present position. In general, therefore, they should be in harmony. This leads us to ask whether too much emphasis has been placed on examples of transition matrices which, by any stretch of imagination, are unlikely to arise in practice." One can indeed establish the compatibility of all the properties outlined above by restricting the domain of mobility matrices. For instance, it is possible to devise mobility indices which satisfy all of Shorrocks' axiorns on the subdomain of mobility matrices with maximal (or, more generally, quasi-maximal) diagonals (Shorrocks, 1978 and Bhattacharya, 1994). Nevertheless, the usefulness of such restrictions is suspect with regard to both theoretical and empirical perspectives (cf. Conlisk, 1990).

41. Denoting the proportion of the individuals with income level $x_{i}$ in the (P-induced) expected income distribution of period $t-1$ by $\pi_{i}(t-1)$ we must have $\pi(t)=\pi(t-1) P$ (where $\left.\pi(t-1)=\left(\pi_{1}(t-1), \ldots, \pi_{m}(t-1)\right)\right)$ for all $t \geq 1$.

42. See Kanbur and Stiglitz (1986) and Kanbur and Stromberg (1988).

43. The proof of this claim is simple and is illustrative of the structure brought in by monotone mobility matrices. Observe first that $P$ is monotone iff $T^{-1} P T \geq 0$ where $T=\left[t_{i j}\right] \in R^{\text {ncom }}$ with $t_{i j}=1$ if $i \leq j$, and $t_{i j}=0$ otherwise. Notice also that "expected" income distribution $\pi(t-1)$ is more equal than (stochastically dominates) the distribution $\pi^{*}(t-1)$ iff $\pi(t-1) T \leq \pi^{*}(t-1) T$ and $P$ stochastically dominates $Q$ iff $P T \leq Q T$. But then, given that $P$ is monotonic, if $\pi(t-1)$ is more equal than $\pi^{*}(t-1)$, and $P$ stochastically dominates $Q$,

$$
(\pi(t-1) P) T-\left(\pi^{*}(t-1) Q\right) T=\left(\pi(t-1)-\pi^{*}(t-1)\right) T\left(T^{-1} P T\right)+\pi^{*}(t-1)(P-Q) T \leq 0
$$

that is, $\pi(t-1) P$ is more equal than $\pi^{*}(t-1) Q$. The converse of the statement is basically trivial.

44. Since $\triangleright_{D}$ can only rank transition matrices with identical steady-state income distributions, it is, in fact, an exchange mobility ordering. Moreover, one can show that it approaches 'income mobility' from the angle of temporal independence (or equality of opportunity). See Dardanoni (1993) for an extensive discussion of these issues.

45. $\nabla_{D}$ is nevertheless a parametric ordering due to its dependence on the discount factor. The probiem of determining the (most general) conditions under which Dardanoni's ordering would rank all monotone and regular transition matrices (with the same equilibrium) for all discount factors, is at the moment open (but see Dardanoni, 1993, Theorem 3, for a partial answer to this question). This problem is in the same spirit as the problem of obtaining a parameter-free characterization of the Mitra-Ok ordering discussed in subsection 3.4.

46. Actually, if the chains under consideration are fractile; i.e., the probability of being in any state at any time is always $1 / m$ then the equal steady-state assumption is automatically met. In this respect, Dardanoni's 
ordering is of considerable use in analyzing interfractile mobility. Moreover, Formby, Smith and Zheng (1995) have shown that Dardanoni's joint-steady state requirement can be relaxed to obtain a mobility ordering that can compare mobility processes that start from any given (not necessarily steady state) distribution $\pi$. Recently, a dual approach is developed by Benabou and Ok (1999) who compare the distributions of expected future incomes (or their present values) under different mobility processes by fixing the income levels $x_{1}, \ldots, x_{m}$ and varying the distribution $\pi$. One advantage of this dual approach is to allow one to view a mobility process as a redistribution policy, and this, in turn, leads to the idea of using tax progressivity indices to assess the extent of mobility as an equalizer of ex ante opportunities.

47. If these assumptions are made, one can standardize the observation period pertaining to the transition matrices. For instance, if $P$ refers to a unit period and $Q$ to $k \geq 2$ many unit periods, by means of (1) and (2), we may adjust $P$ as $P^{k}$ to refer to $k$ periods. But, as demonstrated by Shorrocks (1978), this adjustment is quite problematic. (For instance, what guarantees that the comparison between $P^{k}$ and $Q$, and between $P$ as $Q^{1 / k}$ will yield the same conclusions?)

48. As noted by Dardanoni (1995), however, one may be able to reduce a higher-order Markov chain to a first order one by suitably redefining the boundaries of the income states (cf. Billingsley, 1961).

\section{References}

Abowd, J.M., and D. Card. 1989. "On the Covariance Structure of Eamings and Hours Changes." Econometrica 57: 411-445.

Adelman, I.G. 1958. "A Stochastic Analysis of the Size Distribution of Firms." Joumal of the American Statistical Association 53: 893-904.

Atkinson, A.B. 1970. "On the Measurement of Inequality." Journal of Economic Theory 2: 244-263.

Atkinson, A.B. 1980. "Income Distribution and Inequality of Opportunity." IHS Journal 4: 65-80.

Atkinson, A.B. 1981a. "The Measurement of Economic Mobility. "In P.J. Eggelshoven and L.J. van Gemerden (eds.), Inkommens Verdeling en Openbard Financien. Leiden: Het Spectrum.

Atkinson, A.B. 1981b. "On Intergenerational Income Mobility in Britain." Journal of Post Keynesian Economics 3: $194-218$.

Atkinson, A.B. and F. Bourguignon. 1982. "The Comparison of Multidimensioned Distributions of Economic Starus." Review of Economic Studies 49: 183-201.

Atkinson A.B., F. Bourguignon and C. Morrisson. 1992. Empirical Studies of Earnings Mobility. London: Harwood Academic Publishers.

Atkinson, A.B., A.K. Maynard and C.G. Trinder. 1983. Parents and Children: Incomes in Two Generations. London: Heinemann.

Atoda N. and T. Tachibanaki. 1980. "Earnings Distribution and Inequality Over Time." Discussion Paper 144, Kyoto Institute of Economic Research.

Atoda N. and T. Tachibanaki. 1991. "Earnings Distribution and Inequality Over Time: Education versus Relative Position and Cohort." International Economic Review 32: 475.

Bartholomew, D.J. 1982. Stochastic Models for Social Processes. London: Wiley.

Basmann, R.L., K.J. Hayes and D.J. Slottje. 1991. "The Lorenz Curve and the Mobility Function. "Economics Letters 36: 105-111.

Becker, G.S., and N. Tomes. 1979. "An Equilibrium Theory of Distribution of Income and Intergenerational Inequality." Journal of Political Economy 87: 1153-89.

Becker, G.S., and N. Tomes. 1986. "Human Capital and the Rise and Fall of Families." Joumal of Labor Economics 43: S1-\$39. 
Benabou, R. 1996. "Meritocracy, Redistribution and the Size of the Pie." C.V. Star Center Working paper $96-$ 43, New York University.

Benabou, R. and E.A. Ok. 1999. "Mobility as Progressivity: Ranking Income Processes According to Equality of Opportunity." Mimeo, New York University.

Bhattacharya, K. 1994. "Distribution of Cell Aggregates over Individual Units of n Independent Markov Chains." Mimeo, Indian Statistical Institute.

Bibby, J. 1975. "Methods of Measuring Mobility." Quality and Quantity 9: 107-136.

Bigard, A., Y. Guillotin, C. Lucifora and F. Rappelli. 1995. "An International Comparison of Eamings Mobility: The Case of Italy and France." Paper presented at the Seventh EALE Conference (1995).

Billingsley, P. 1961. Statistical Inference for Markov Processes. New York: Wiley and Sons.

Blackorby, C. and D. Donaldson. 1978. "Measures of Relative Equality and Their Meaning in Terms of Social Welfare." Joumal of Economic Theory 18: 59-80.

Blackorby, C. and D. Donaldson. 1980. "A Theoretical Treatment of Indices of Absolute Inequality." International Economic Review 21: 107-136.

Boudon, R. 1973. Mathematical Structures of Social Mobility. Amsterdam: Elsevier.

Buchinsky, M. and J. Hunt. 1996. "Wage Mobility in the United States." NBER Working Paper 5455.

Chakravarty, S.J. 1984. "Nornative Indices for Measuring Social Mobility." Economics Letters 15: 175-180.

Chakravarty, S.J. 1990. Ethical Social Index Numbers. Berlin: Springer-Verlag.

Chakravarty, S.R. 1984. "On Kullback's Measure of Mobility." Mimeo, Indian Statistical Institute.

Chakravarty, S.J. and B. Dutta. 1987. "A Note on Measures of Distances Between Income Distributions." Journal of Economic Theory 41: 185-189.

Chakravarty, S.J., B. Dutta, and J.A. Weymark 1985. "Ethical Indices of Income Mobility. "Social Choice and Welfare 2: 1-21.

Checchi, D., A. Ichino and A. Rustichini. 1995. "Talis Pater Talis Filius? Intergenerational Mobility and the Transmission of Talent, Education and Wealth: Comparative Evidence from Italy and the US." Mimeo, Universita Bocconi.

Coleman, J.S. 1964. Introduction to Mathematical Sociology: London: Macmillan.

Conlisk, J. 1974. "Can Equalization of Opportunity Reduce Social Mobility?" American Economic Review 64: $80-90$.

Conlisk, J. 1985. "Comparative Statics for Markov Chains." Journal of Economic Dynamics and Control 9: 139-151.

Conlisk, J. 1989. "Ranking Mobility Matrices." Economics Letters 29: 231-235.

Conlisk, J. 1990. "Monotone Mobility Matrices." Journal of Mathematical Sociology 15: 173-191.

Cowell, F.A. 1985. "Measures of Distributional Change: An Axiomatic Approach." Review of Economic Studies, 52: 135-151.

Creedy, J. 1985. Dynamics of Income Distribution. Oxford: Basil Blackwell.

Creedy, J. 1992. "Increasing the Time Period in the Measurement of Inequality. "In D.J. Slottje (ed.), Research on Economic Inequality, Vol. 2. Greenwich, CN: JAI Press.

Dagum, C. 1980. "Inequality Measures Between Income Distributions with Applications." Econometrica 48: 1791-1803.

Dardanoni, V. 1991. "On the Lorenz Curve Ordering of Discounted Streams of Income." Bulletin of Economic Research 43: 293-296.

Dardanoni, V. 1993. "Measuring Social Mobility." Journal of Economic Theory 61: 372-394.

Dardanoni,Y. 1995. "Monotone Mobility Matrices and Income Distribution Dynamics." Social Choice and Welfare 12: 181-192. 
Dardanoni, V and A. Forcina. 1993. "You'd Better Have A Rich Father: Testing Monotone Mobility Matrices." Mimeo, University of Palermo.

Department of Employment, United Kingdom. 1973. "Low Pay and Changes in Earnings." Employment Gazette April: $335-348$.

Ebert, U. 1984. "Measures of Distance Between Income Distributions." Journal of Economic Theory 32: 266-274.

Ebert, U. 1988. "A Family of Aggregative Compromise Inequality Measures." International Economic Review 29: 363-376.

Eichhorn, W. and W. Gehrig. 1982. "Measurement of Inequality in Economics." In B. Korte (ed.), Modem Applied Mathematics - Optimization and Operations Research. Amsterdam: North-Holland.

Erickson, R. and J.H. Goldthorpe 1982. The Constant Flux. A Study of Class Mobility in Industrial Societies. Oxford: Clarendon Press.

Fields, G.S. and E.A. Ok. 1996. "The Meaning and Measurement of Income Mobility." Joumal of Economic Theory 71: 349-377.

Fields, G.S. and E.A. Ok. 1999. "Measuring Movement of Incomes." Mimeo, NYU (forthcoming in Economica).

Formby, J., J. Smith and B. Zheng. 1995. "Economic Growth, Welfare and the Measurement of Social Mobility." Mimeo, University of Colorado-Denver.

Foster, J.E. 1985. "Inequality Measurement." In P. Young (ed.), Fair Allocations, Vol. 33. Providence, RI: AMS Proceedings of Symposia in Applied Mathematics.

Foster, I.E., J. Greer and E. Thorbecke. 1984. "A Class of Poverty Measures." Econometrica 52: 761-765.

Foster, J.E. and Y. Jin. 1994. "Poverty Orderings for the Dalton Utility-Gap Measures." Mimeo, Vanderbilt University.

Friedman. M. 1962. Capitalism and Freedom. Chicago: University of Chicago Press.

Geweke, J., R. C. Marshall and G.A. Zarkin. 1986. "Mobility Indices in Continuous Time Markov Chains." Econometrica 54: 1407-1424.

Goldberger, A. 1989. "Economic and Mechanical Models of Intergenerational Transmission." American Economic Review 79: 504-513.

Gottschalk, P. 1982. "Earnings Mobility: Permanent Change or Transitory Fluctuations?" Review of Economics and Statistics 64: 450-456.

Gottschalk, P. 1997. "Inequality, Income Growth and Mobility: The Basic Facts." Journal of Economic Perspectives 11:21-40.

Gottschalk, P. and E. Spolaore. 1998. "On the Evaluation of Economic Mobility." Mimeo, Boston College.

Hart, P.E. 1976a. "The Dynamics of Eamings: 1963-1973." Economic Joumal 86: 335-346.

Hart, P.E. 1976b. "The Dynamics of Earnings: 1963-1973. " Journal of the Royal Statistical Society, Series A, 139: $108-125$.

Hart, P.E. 1981. "The Statics and Dynamics of Income Distributions: A Survey." In N.A. Klevmarken and J.A. Lybeck (eds.), The Statics and Dynamics of Income. Tieto: Clevedon.

Jarvis, S. and S. Jenkins 1996. "Changing Places: Income Mobility and Poverty Dynamics in Britain." Working paper 96-19, ESRC Research Centre on Micro-social Change, University of Essex.

Jenkins, S. 1987. "Snapshots versus Movies: 'Lifecycle Biases' and the Estimation of Intergenerational Earnings Inheritance." European Economic Review 31: 1149-1158.

Kanbur, S.M.R. and J. Stiglitz. 1986. "Intergenerational Mobility and Dynastic Inequality. " Mimeo, Princeton University.

Kanbur, S.M.R. and J-O. Stromberg. 1988. "Income Transitions and Income Distributions." Joumal of Economic Theory 45: 408-416. 
Karcher, T., P. Moyes and A. Trannoy. 1995. "The Stochastic Dominance Orderings of Income Distributions Over Time I." In W. Barnett et al. (eds.), Social Choice, Welfare and Ethics, Proceedings of the 8th International Symposium in Economic Theory and Econometrics. Cambridge: Cambridge University Press. Kearl, J.R. and C.L. Pope 1984. "Mobility and Distribution." Review of Economics and Statistics 66: $192-199$. Keilson J. and A. Ketser 1977. "Monotone Matrices and Monotone Markov Chains." Stochastic Processes and Their Applications 5: 231-241.

King, M.A. 1983. "An Index of Inequality: With Applications to Horizontal Equity and Social Mobility." Econometrica 51: 99-115.

Klevmarken, A. and J.A. Lybeck (eds.). 1981. The Statics and Dynamics of Income. Tieto: Clevedon.

Kolm, S.C. 1969. "The Optimal Production of Social Justice." In J. Margolis and H. Guitton (eds.), Public Economics. London: Macmillan.

Kolm, S.C. 1976. "Unequal Inequalities I." Joumal of Economic Theory 12: 416-442.

Lambert, P.J. 1993. The Distribution and Redistribution of Income: A Mathematical Analysis. Manchester: Manchester University Press.

Lillard, L.A. 1977. "Inequality: Earnings vs. Human Wealth." American Economic Review 67: 42-53.

Lillard, L.A., and R.J. Willis. 1978. "Dynamic Aspects of Earnings Mobility." Econometrica 46: 985-1012.

Loury, G.C. 1981. "Intergenerational Transfers and the Distribution of Earnings." Econometrica 49: 843-867.

Maasoumi, E. 1996. "On Mobility." Mimeo, Southern Methodist University.

Maasoumi E, and S. Zandvakili. 1986. "A Class of Generalized Measures of Mobility with Applications." Economics Letters 22: 97-102.

Maasoumi E. and S. Zandvakili. 1990. "Generalized Entropy Measures of Mobility for Different Sexes and Income Levels." Journal of Econometrics 43: 121-133.

Markandya, A. 1982a. "Intergenerational Exchange Mobility and Economic Welfare." European Economic Review 17: 301-324.

Markandya, A. 1982b. "The Measurement of Earnings Mobility Among Occupational Groups." Scottish Journal of Political Economy 29: 75-88.

Markandya, A. 1982c. "The Welfare Measurement of Changes in Economic Mobility." Economica 51: $457-471$.

McCall, J.J. 1973. Income Mobility, Racial Discrimination and Economic Growth. Lexington: MA: Heath.

Mitra T. and E.A. Ok. 1998. "The Measurement of Income Mobility: A Partial Ordering Approach." Economic Theory 12: 77-102.

Moffitt, R.A. and P. Gottschalk. 1995. "Trends in the Autocovariance Structure of Eamings in the U.S: 19691987." Working Paper, The Johns Hopkins University.

Prais, S.J. 1955. "Measuring Social Mobility." Joumal of the Royal Statistical Society, Series A, 118: 56-66. Schiller, B. 1977. "Relative Eamings Mobility in the U.S.." American Economic Review December: 926-941. Scott, C.D. and J.A. Litchfield. 1994. "Inequality, Mobility and the Determinants of Income Among the Rural Poor in Chile, 1968-1986." Working Paper No. 53, Development Economics Research Programme, London School of Economics.

Sen, A.K. 1973. On Economic Inequality. Oxford: Oxford University Press.

Sen, A.K. 1977. "Personal Utilities and Public Judgements: Or What's Wrong with Welfare Economics?" Economic Journal 89: 537-558.

Sen, A.K. 1976. "Poverty: An Ordinal Approach to Measurement." Econometrica 45: 219-231.

Shorrocks, A.F. 1976. "Income Mobility and the Markov Assumption." Economic Joumal 46: 566-578.

Shorrocks, A.F. 1978a. "The Measurement of Mobility." Econometrica 46: 1013-1024.

Shorrocks, A.F. 1978b. "Income Inequality and Income Mobility." Joumal of Economic Theory 46: 376-393.

Shorrocks, A.F. 1981. "Income Stability in the United States." In N.A. Klevmarken and J.A. Lybeck (eds.), The Statics and Dynamics of Income. Tieto: Clevedon. 
Shorrocks, A.F. 1982. "On the Distance Between Income Distributions." Econometrica 50: 1337-1339.

Shorrocks, A.F. 1993. "On the Hart Measure of Income Mobility." In M. Casson and J. Creedy (eds.), Industrial Concentration and Economic Inequality. Cambridge: Edward Elgar.

Silber, J. 1995. "Horizontal Inequity, the Gini Index and the Measurement of Distributional Change." In C. Dagum and A Lemmi (eds.), Income Distribution, Social Welfore, Inequality and Poverty, Research on Economic Inequality, Vol. VI. Greenwich, CN: JAI Press.

Silber, J. and Z.M. Berrebi. 1988. "Distance Functions and the Comparison of Development Levels." Economics Letters 27: 195-200.

Slesnick, D.T. 1986. "Welfare Distributional Change and the Measurement of Social Mobility." Review of Economics and Statistics LXVIII(4): 586-593.

Solon, G.R. 1992. "Intergenerational Income Mobility in the United States." American Economic Review 82: 393-408.

Sommers, P.M. and J. Conlisk. 1978. "Eigenvalue Immobility Measures for Markov Chains." Journal of Mathematical Sociology 6: 253-276.

Thatcher, A.R. 1971. "Year-to-year Variations in the Earnings of Individuals." Joumal of Royal Statistical Sociery, Series A, 134: 374-382.

Theil, H. 1972. Statistical Decomposition Analysis. Amsterdam: North-Holland.

Watts, H. 1968. "An Economic Definition of Poverty." In D.P. Moynihan (ed.), On Understanding Poverty. New York: Basic Books.

Zheng, B. 1993. "An Axiomatic Characterization of the Watts Poverty Index." Economics Letters 42: 81-86. Zimmerman, D.J. 1992. "Regression Toward Mediocrity in Economic Stature." American Economic Review 82: 409-429. 